\title{
Nuisibilité et seuils de nuisibilité des mauvaises herbes dans une culture annuelle: situation de concurrence bispécifique
}

\author{
J.P. Caussanel \\ INRA, Laboratoire de Malherbologie, BV 1540, 21034 Dijon Cedex, France
}

(reçu le 24-4-1988, accepté le 18-12-1988)

\begin{abstract}
Résumé - La nuisibilité des mauvaises herbes dans une culture annuelle est à relier principalement à leurs effets négatifs sur la croissance et le développement de la plante cultivée. En termes d'interactions biologiques, les effets mesurés traduisent les résultats de la concurrence entre mauvaises herbes et plante cultivée. Ils sont causés par divers processus biologiques comme la compétition, l'allélopathie ou tout autre processus d'exploitation. Dans cet article, les seuils biologiques et économiques de nuisibilité sont définis dans une culture annuelle en faisant référence à une espèce adventice dominante dans la culture. La densité de l'adventice est généralement utilisée pour exprimer ces seuils de nuisibilité et pour établir des courbes de régression du rendement. Cependant, l'analyse des mécanismes de la concurrence due aux mauvaises herbes dans les cultures montre que la détermination du seuil biologique de nuisibilité est améliorée en prenant en considération d'autres facteurs démographiques que la densité comme la structure temporelle ou la structure spatiale des populations végétales en présence. Pour chaque espèce adventice, la connaissance de ces données est nécessaire afin d'estimer des seuils économiques de nuisibilité et d'établir des prévisions de risques à plus ou moins long terme.
\end{abstract}

mauvaises herbes - cultures annuelles - compétition - pertes de rendement - évaluation des dégâts - prévisions de risques

\begin{abstract}
Summary - Injurious effects of weeds and weed thresholds in an annual arable crop: interference between two species. Injurious effects of weeds in an annual arable crop are mainly related to their negative influence on crop growth and development. When such negative effects are measured among all different biological interactions, the following biological process may be implicated: competition, allelopathy or any other process of exploitation. In this paper, biological and economic thresholds are assessed in an annual arable crop in relation to one main weed species. Weed density is generally used to express these thresholds and to relate crop loss with weed infestation. However, analysis at the field scale of weed competition mechanisms in an annual arable crop shows that biological threshold assessment is enhanced by taking into account other demographic factors such as weed period or weed distribution. Knowledge of these data for every weed species is needed to assess economic thresholds and to make short and long-range risk forecasting.
\end{abstract}

weeds - annual crops - competition - crop losses - damage assessment - risk forecasting

\section{Introduction}

Dans une agriculture moderne, le désherbage chimique des cultures principales est devenu une opération de routine parmi les techniques culturales. Différentes raisons socio-économiques plaident aujourd'hui pour une rationalisation accrue du désherbage afin d'éviter au maximum les traitements inutiles ou superflus.

A l'échelon d'une parcelle ou d'une exploitation, tout programme de désherbage devrait être envisagé en fonction des risques de nuisibilité que les mauvaises herbes font courir aux plantes cultivées et des dégâts potentiels sur les produits récoltés. Or, un programme de désherbage s'inscrit dans un itinéraire technique de culture et se prévoit en termes économiques, alors que la nuisibilité due aux mauvaises herbes, dite aussi «nuisibilité adventice», s'évalue à partir de données expérimentales mesurées en biologie des populations dans un milieu artificiellement crée par l'homme : l'agroécosystème. 
De nombreuses expérimentations ont établi des liaisons entre la perte de rendement d'une culture et divers facteurs structuraux qui règlent la biologie des populations de mauvaises herbes. Mais aucune généralisation ne permet encore, au stade actuel de nos connaissances, de présenter des seuils de nuisibilité adventice fiables et utiles à la pratique du désherbage, même dans les cultures annuelles, où la majorité des recherches ont été menées. Une telle généralisation est-elle possible et souhaitable?

Dans cet article, les définitions et concepts communément admis au sujet de la nuisibilité et de la concurrence des mauvaises herbes sont présentés. Puis, à l'aide de quelques exemples, les pertes de rendement dues à la nuisibilité adventice dans une culture annuelle sont analysées et reliées à l'économie du désherbage.

\section{Nuisibilité due aux mauvaises herbes dans une culture annuelle}

\section{Nuisibilité due aux mauvaises herbes}

Appliqué aux mauvaises herbes, le concept de nuisibilité englobe deux sortes d'effets :

- la nuisibilité due à la flore potentielle, dont il faudrait tenir compte si, pour chaque espèce, chacun des organes de multiplication conservés dans le sol à l'état de repos végétatif (semences, bulbes, tubercules, etc..) donnait un individu à la levée. En fait, ce risque doit être réduit dans les prévisions : en effet, avec un potentiel semencier de l'ordre de 4000 semences viables par $\mathrm{m}^{2}$ et si l'on admet que les levées au champ représentent généralement entre $5 \%$ et $10 \%$ du nombre de semiences enfouies, les infestations prévisibles d'une culture représentent 200 à 400 adventices par $\mathrm{m}^{2}$ (Roberts, 1981; Barralis \& Chadoeuf, 1987);

- la nuisibilité due à la flore réelle, c'est-à-dire aux plantes qui lèvent réellement au cours du cycle de la culture (Fig. 1).

Chaque espèce adventice possède sa propre nuisibilité (nuisibilité spécifique) qui contribue à la nuisibilité globale du peuplement adventice dans des conditions d'offre environnementale définies.

Lorsque la nuisibilité due à la flore adventice réelle n'est prise en compte que par ses effets indésirables sur le produit récolté, cette nuisibilité est dite primaire. Si les dommages dus à l'action conjuguée de la flore réelle et de la flore potentielle s'étendent aussi à la capacité ultérieure de production, soit au niveau de la parcelle (accroissement du potentiel semencier du sol notamment), soit au niveau de l'exploitation agricole (création et multiplication de foyers d'infestation,

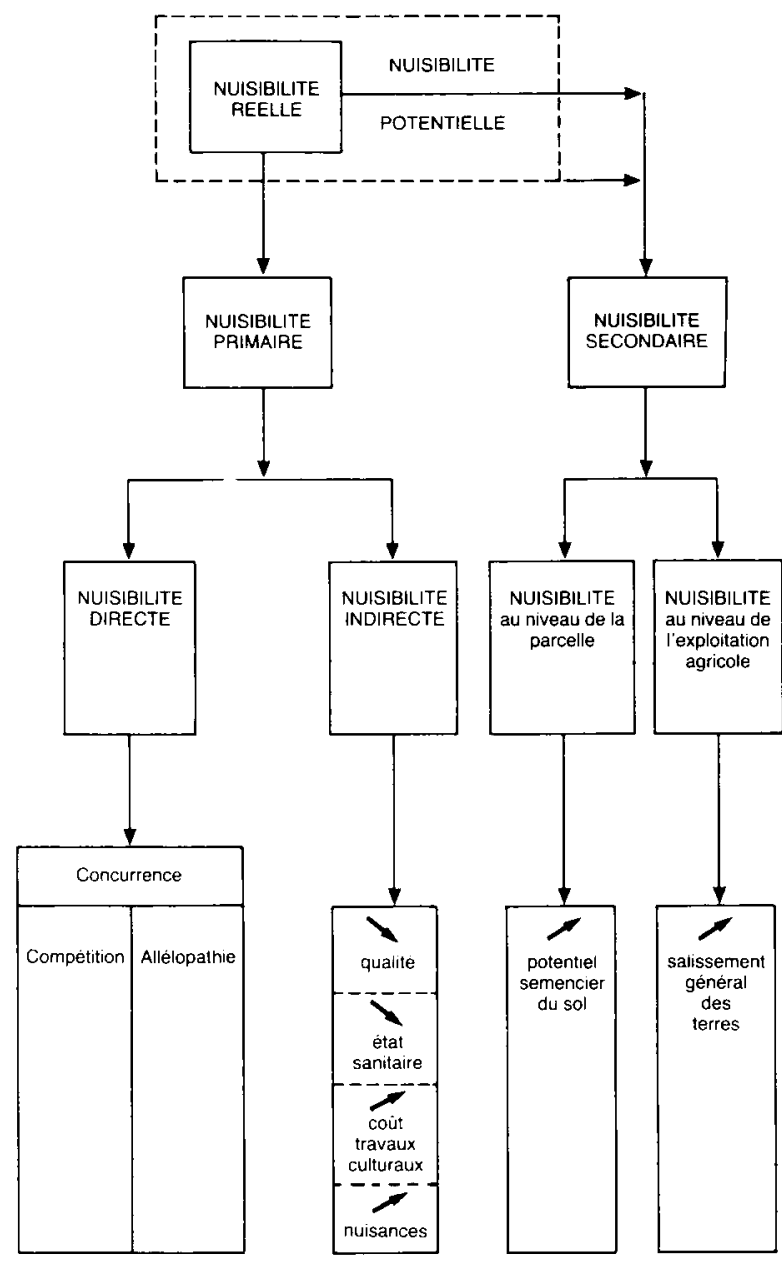

Fig. 1. Types de nuisibilité des mauvaises herbes dans les cultures (d'après Chiarappa, 1981).

contamination du sol ou du matériel végétal, nuisances et pollution), la nuisibilité est qualifiée de secondaire.

En voyant les mauvaises herbes d'une culture gêner la croissance de la plante cultivée et entraver son développement jusqu'à l'étouffer parfois complètement, il est clair que la nuisibilité primaire s'exerce sur la quantité du produit récolté. Mais elle modifie également sa qualité. Par la diminution quantitative de production que leur présence entraîne, les mauvaises herbes manifestent directement leur nuisibilité : cette nuisibilité est dite directe. Par opposition, tous les autres effets indésirables des mauvaises herbes sur l'élévation du coût de la production du produit commercialisable sont regroupés sous le nom de nuisibilité indirecte. Sans qu'il y ait nécessairement réduction quantitative du rendement, la nuisibilité indirecte peut porter soit sur l'abaissement de la qualité et par suite de la valeur commerciale du produit récolté, soit sur la diminution de l'état sanitaire de la culture (plantes adventices réservoirs ou hôtes de divers parasites), soit sur l'augmentation du coût des travaux culturaux, 
avant, pendant ou après récolte, soit encore sur l'élévation des risques de nuisances (toxicité sur les êtres vivants, gêne pour les activités humaines ou dangers pour l'environnement). Les effets indésirables des mauvaises herbes dans les cultures ont été illustrés par de nombreux exemples dans la littérature (King, 1966; Longchamp, 1977 a, b; Elliott, 1980; Koch et Walter, 1983).

Interactions biologiques entre mauvaises herbes et plantes cultivées

La nuisibilité directe due à la flore adventice, nuisibilité dont les effets négatifs sont mesurés sur le rendement du produit récolté, résulte de diverses actions dépressives auxquelles sont soumises les plantes cultivées pendant leur cycle végétatif de la part des mauvaises herbes qui les entourent.

Dans un peuplement végétal, la présence d'une plante change l'environnement des plantes voisines, dont elle peut altérer la croissance et la forme. De telles «intertérences», selon la dénomination de Harper (1977), comprenaient dans la définition d'origine les effets dus aussi bien à la consommation de ressources en disponibilités limitées qu'à la production de toxines ou qu'aux changements environnementaux qu'apporte la protection contre le vent, ou que crée le comportement des prédateurs.

Le terme anglosaxon «interférences» est actuellement usité pour décrire les seuls effets d'action directe entre organismes (Begon et al., 1987). Les «interactions biologiques» entre végétaux (dénommées aussi selon les auteurs "actions biologiques réciproques", "effets de voisinage», "effets de groupe», «relations sociales», etc.) se manifestent au niveau de l'individu (déviations plastiques sur tout ou partie de cet individu) et au niveau du peuplement (évolution du peuplement par mortalité d'individus). Elles se définissent soit par leur nature soit par leurs processus à partir de leurs manifestations (Jacquard, 1968). Les définitions fondées sur la nature des interactions sont établies à partir des effets observés sur chaque organisme participant (Odum, 1959). Celles qui prennent les processus en considération s'appuient sur les causes qui sont à l'origine des interactions (Milne, 1961).

En première approche, la classification la plus cohérente des interactions biologiques qui existent entre les organismes ou deux ensembles d'organismes vivants est basée sur leur nature (Malcolm, 1966; Jacquard, 1968, 1975; Lemée, 1978; Radosevich et Holt, 1984). La description est fondée sur les modifications de croissance et de développement qu'apporte, au niveau de cha- cun des deux éléments du groupe, le passage de l'état isolé (situation d'،isolement" ou de "séparation" ou d'céloignement») à l'état associé (situation d' «association» ou de "rencontre» ou de «voisinage»).

Dans un peuplement monospécifique, l'individu $A$ est amené à partager avec un ou plusieurs autres individus $A$ la même cellule de ressources aux conditions d'offre environnementale définies. Sa production individuelle dans cette culture pure peut être mesurée et symbolisée par y a/a. Dans un peuplement bispécifique, en culture associée, la production individuelle de l'individu $A$ en présence de plusieurs individus de l'espèce $B$ est symbolisée par y $a / b$. De même, y $b / b$ et $y b / a$ sont mesurables. Trois signes suffisent à définir le sens de la modification:

+ : augmentation de croissance et de développement

- : réduction de croissance et de développement ou impossibilité de développement

0 : croissance et développement non modifiés

En affectant l'un de ces signes à chaque organisme, deux signes sont nécessaires pour caractériser la situation à l'état isolé des deux organismes, et deux autres pour caractériser la situation à l'état associé. Quatre signes servent donc à décrire comparativement les deux situations, ce qui conduit à $3^{4}=81$ interactions théoriquement possibles ( 9 types d'،effets de rencontre» et 9 types d'ceffets de séparation»). En fait, une dizaine seulement ont un sens biologique (Malcolm, 1966). A partir de la situation logique où le signe 0 représente une croissance et un développement "normaux» pour chacun des deux organismes dans des conditions données d'offre environnementale, toute situation d'association peut recevoir une dénomination, selon le sens de la modification de croissance et de développement pour chaque organisme (Tableau I). Certaines de ces dénominations ne sont que des propositions et leur emploi doit être fait avec quelques réserves d'ordre conceptuel ou d'ordre expérimental dans le cas de groupements végétaux (Jacquard, 1980).

\section{Concurrence des mauvaises herbes dans une culture annuelle}

\section{Situation de concurrence dans une culture annuelle}

A cause de la manifestation d'effets dépressifs qu'implique la notion de "concurrence», ce terme est généralement usité pour toute interaction biologique où deux organismes à croissance et développement normaux à l'état isolé (00) sont 
Tableau I. Interactions biologiques entre deux organismes ou populations A et B (d'après Malcom, 1966; Jacquard, $1968,1975)$.

\begin{tabular}{|c|c|c|c|c|c|c|}
\hline \multicolumn{2}{|c|}{ Etat isolé } & \multicolumn{2}{|c|}{ Etat associé } & \multicolumn{2}{|c|}{$\begin{array}{l}\text { Nature des effets au niveau } \\
\text { des éléments du groupe }\end{array}$} & \multirow[t]{2}{*}{$\begin{array}{l}\text { Nom de la } \\
\text { situation }\end{array}$} \\
\hline$A$ & $B$ & $A$ & $B$ & $y a / b-y a / a$ & $y b / a-y b / b$ & \\
\hline 0 & 0 & 0 & 0 & 0 & 0 & $\begin{array}{l}\text { Neutralisme ou } \\
\text { Indépendance }\end{array}$ \\
\hline 0 & 0 & + & + & + & + & $\begin{array}{l}\text { Coopération ou } \\
\text { Mutualisme }\end{array}$ \\
\hline- & - & $\underline{0}$ & \begin{aligned} 0 \\
\hdashline\end{aligned} & + & + & Symbiose \\
\hline 0 & 0 & 0 & + & 0 & + & Commensalisme \\
\hline 0 & 0 & - & - & - & - & \\
\hline 0 & 0 & - & 0 & - & 0 & Concurrence \\
\hline 0 & 0 & - & + & - & + & \\
\hline 0 & - & - & 0 & - & + & Parasitisme \\
\hline
\end{tabular}

y $a / a$ : production de l'organisme $A$ en présence de lui-même. $y$ a/b : production de l'organisme $A$ en présence de $B$. y $b / b:$ production de l'organisme $B$ en présence de lui-même. y b/a : production de l'organisme $B$ en présence de $A$. L-- $_{-}$: processus biologique exprimant la dépendance de l'un des deux organismes au moins par rapporté à l'autre.

déprimés, au moins pour l'un d'entre eux, à l'état associé $(--,-0,-+)$. Selon une définition classique chez les végétaux (Bleasdale, 1959), deux plantes entrent en situation de concurrence lorsque la croissance de l'une d'entre elles ou celle des deux est réduite ou que leur forme est modifiée, comparativement à la croissance ou à la forme qu'elles ont isolément. C'est là une définition qui repose sur les manifestations d'effets quantitativement mesurables. D'autres auteurs préfèrent caractériser le phénomène de concurrence par ses causes (modifications de l'environnement local d'un individu par d'autres individus adjacents, Milthorpe 1961; Guy, 1965) ou préciser sa nature à l'aide de termes plus spécifiques (comme "contraintes de densité", «interférences", "effets de voisinage», Harper, 1977).

Dans les peuplements végétaux cultivés, la concurrence est une situation courante : dans une étude expérimentale de relations entre plantes fourragères constituées de graminées et de légumineuses (Caputa, 1948), la situation de concurrence, mesurée par un effet dépressif sur l'un au moins des constituants de l'association, a représenté $91 \%$ des combinaisons étudiées (Jacquard, 1968). En ce qui concerne les relations entre plantes cultivées et adventices, il est logique de relier la nuisibilité directe des mauvaises herbes aux effets de concurrence diminuant la production de la plante cultivée par rapport à la production obtenue en l'absence de mauvaises herbes. II est plus malaisé de préciser cette interaction par la nature de l'effet sur la mauvaise herbe, en raison de la référence à une culture pure adventice qui n'est pas une situation agronomique.

Dans une culture annuelle qui ne comporte qu'une seule espèce cultivée et plusieurs espèces adventices, il existe quatre situations de concurrence (Caussanel, 1979) :

a) intraspécifique ou intravariétale entre les individus appartenant à l'espèce ou à la variété cultivée;

b) intraspécifique entre les individus appartenant à la même espèce adventice;

c) interspécifique entre les individus appartenant à plusieurs espèces adventices;

d) interspécifique entre les individus appartenant à l'espèce (ou à la variété) cultivée, et à une ou plusieurs espèces adventices.

Dans les peuplements plurispécifiques (exemple de l'écosystème prairial), l'étude de la dynamique des relations de concurrence interspécifiques, interpopulations et intrapopulations conduit à l'utilisation de modèles prévisionnels, statiques et dynamiques, et de modèles de simulation, dynamiques et mécanistes (Jacquard, 1982). Certains types de modèles ont été appliqués aux relations de concurrence interspécifique dans un peuplement plurispécifique comprenant des mauvaises herbes, mais la plupart 
d'entre eux restent appliqués aux relations de concurrence bispécifique entre une espèce adventice et une espèce cultivée (de Wit, 1960; Trenbath, 1976).

Pour être complète, l'analyse des effets de concurrence bispécifique entre une plante cultivée et une mauvaise herbe nécessite de faire appel aux processus qui expliquent les diverses interactions inclues dans le terme général de concurrence (Tableau I).

\section{Compétition due aux mauvaises herbes}

La compétition se définit comme la concurrence qui s'établit entre plusieurs organismes pour une même source d'énergie ou de matière lorsque la demande est en excès sur les disponibilités (Lemée, 1967). Quelles sources d'énergie ou de matière peuvent se comporter en "facteurs limitants du milieu»? La lumière, les éléments nutritifs du sol (tout particulièrement l'azote) et l'humidité du sol sont les plus connus; plusieurs mises au point sur leur rôle dans les mécanismes de la compétition ont été présentées (Caussanel et Barralis, 1973; Glauninger \& Holzner, 1982). Certaines mauvaises herbes comme, par exemple, la folle avoine (Avena fatua L.) présentent de nombreux avantages compétitifs sur les céréales cultivées (Chancellor et Peters, 1976; Thurston, 1982) : la perte de rendement que subit la céréale à la récolte peut être directement reliée à des caractères biologiques ou physiologiques qui assurent le succès de la folle avoine dans la compétition pour la lumière (Wimschneider et Bachthaler, 1979) ou les éléments nutritifs (Koch, 1967). Des plantules de folle avoine provenant de graines lourdes (Peters, 1985) ou des espèces de folles avoines à racines profondes (Ellern et al., 1970) sont également favorisées dans leur "compétition pour l'espace», notamment au cours des premiers stades de développement. Mais la compétition pour l'espace ne représente-t-elle pas la résultante dans le temps de la compétition pour chacun des facteurs limitants du milieu, à l'intérieur d'une cellule de ressources donnée?

II n'est pas possible sur le terrain de faire la distinction entre le processus biologique que représente la compétition et la situation biométrique mesurant une réduction de croissance pour au moins un des deux éléments du groupe. C'est pourquoi, selon les auteurs et selon l'objet de leur étude, la compétition peut être définie soit par le fait qu'un individu profite aux dépens de l'autre (situation + - à l'état associé, Jacquard, 1975); soit par le fait que les deux individus sont déprimés (situation - - à l'état associé, Malcom, 1966; Radosevich et Holt, 1984). Le cas particu- lier où l'individu le plus agressif n'est pas affecté (situation 0 - à l'état associé) ne peut pas non plus être exclu de la définition. De ce point de vue, qui confond la cause de l'effet avec sa nature, définie par la mesure expérimentale, le terme "compétition" est donné par certains auteurs comme synonyme du terme "concurrence».

\section{Allélopathie due aux mauvaises herbes}

Le terme d'allélopathie désigne l'émission ou la libération par une espèce végétale ou par l'un de ses organes, vivants ou morts, de substances organiques toxiques entraînant l'inhibition de la croissance de végétaux se développant au voisinage de cette espèce ou lui succédant sur le même terrain (Borner, 1968; Whittaker, 1970; Rice, 1974; Putnam, 1985). Par cette définition, les interactions chimiques entre végétaux comprennent celles qui s'exercent soit directement entre les plantes (cholines), soit indirectement par l'intermédiaire de microorganismes (marasmines et phytoncides) pendant la vie active des végétaux et au cours de la décomposition de leurs résidus; le terme d'antibiose s'applique plus spécifiquement aux interactions chimiques entre microorganismes.

Les phénomènes d'allélopathie ont été surtout décrits et analysés dans certaines formations végétales comme la garrigue méditerranéenne (Deleuil, 1950), le chaparral californien (Muller, 1966), la prairie pauvre de succession secondaire dans I'Oklahoma (Rice, 1971) ou au Japon (Numata, 1956), la steppe arbustive ukrainienne (Grodzinsky et Gaidamak, 1971). Soupçonnés d'exister également dans les peuplements végétaux cultivés (Martin et Rademacher, 1960; Tukey, 1969; Putnam et Duke, 1978; Grodzinsky, 1982), les phénomènes d'allélopathie ont été démontrés expérimentalement in vitro, dans des essais biologiques, où des substances organiques émises, exsudées ou excrétées par une plante se sont révélées inhibitrices de croissance à partir d'une certaine dose. Parmi les plantes responsables de tels effets, figurent des plantes cultivées comme l'avoine (Martin, 1957; Fay et Duke, 1977), le tournesol (Wilson et Rice, 1968; Leather, 1983), le concombre (Putnam et Duke, 1974), le noyer (Bode, 1958; Coder, 1983), ainsi que des plantes adventices comme Avena fatua L. (Schumacher et al., 1983), Chenopodium album L. (Caussanel et Kunetsch, 1979) et d'autres encore (Tauscher, 1988). Toujours dans des essais biologiques, des extraits aqueux de résidus de paille de céréale se sont montrés phytotoxiques soit vis-à-vis de plantules de blé (Guenzi et al., 1967), soit vis-à-vis de mauvaises herbes se développant l'année suivant la culture 
de cette céréale, dans des systèmes de techniques culturales réduites (Liebl \& Worsham, 1983). Les acides phénoliques identifiés dans les extraits étaient présents à des concentrations suffisantes pour être éventuellement responsables d'effets inhibiteurs sur le terrain en fonction de leur localisation (Liebl \& Worsham, 1983) ou des conditions pédoclimatiques de la culture (Burgos Leon, 1975). L'émission par certaines espèces de substances inhibitrices volatiles, souvent aromatiques, a également été démontrée (Muller et al., 1968). Champignons et bactéries libèrent aussi des substances chimiques inhibitrices qui pourront être utilisées par l'industrie phytosanitaire soit après synthèse, soit après production par manipulation cellulaire ou génétique, soit encore après modification chimique des sites actifs (Duke, 1986).

II arrive que les recherches menées sur les possibilités allélopathiques d'une espèce adventice (par exemple Agropyron repens (L.) P. Beauv.: Osvald, 1948; Grummer, 1961; Welbank, 1963; Gabor et Veatch, 1981) ou sur la réalité d'effets allélopathiques entre une plante adventice et une plante cultivée (par exemple Camelina sativa (L.) Crantz et Linum usitatissimum : Grummer et Beyer, 1960; Balschun et Jacob, 1972; Kranz et Jacob, 1977; Lovett et Jackson, 1980), aboutissent à des causes explicatives différentes. La séparation des effets d'allélopathie de ceux de compétition demande, pour être démontrée, que plusieurs preuves expérimentales soient apportées (Fuerst et Putnam, 1983) :

- établir l'existence d'effets dépressifs sur l'une des deux plantes en identifiant les symptômes et en quantifiant si possible les effets;

- isoler, mettre en évidence au moyen d'essais biologiques, caractériser et synthétiser la substance phytotoxique;

- reproduire les effets dépressifs observés en appliquant cette substance dans les conditions naturelles sur la plante sensible;

- relier les effets dépressifs observés aux symptômes identifiés en analysant l'émission, le transport et l'absorption de la substance toxique.

Actuellement, l'écologie de l'émission des substances inhibitrices impliquées dans les interactions chimiques entre végétaux est encore trop peu étudiée pour ne pas laisser planer le doute sur l'influence réelle des effets allélopathiques dus aux mauvaises herbes dans les cultures annuelles.

\section{Parasitisme, exploitation et antagonisme}

L'existence de mauvaises herbes parasites comme la cuscute des champs (Cuscuta campestris Yuncker) ou l'orobanche crénelée (Oro- banche crenata Forsk.), pour ne citer que les plus nuisibles, demande à définir la notion de parasitisme. C'est le mode de vie d'un organisme qui vit aux dépens de l'hôte avec lequel il est associé, par prélèvement direct de molécules produites au cours de l'activité métabolique de l'hôte; le prélèvement est assuré par des organes d'absorption, appelés suçoirs ou «haustoria». La description des mauvaises herbes parasites des terres arables est basée sur trois critères (Musselman, 1982) :

- le degré de dépendance vis-à-vis de l'hôte (parasites obligatoires ou facultatifs),

- la localisation des haustoria sur l'hôte (parasites sur tiges et sur racines),

- la présence ou l'absence de chlorophylle chez le parasite (hémiparasites ou holoparasites).

A l'état isolé, c'est-à-dire en l'absence d'un hôte, les plantes parasites sont réduites dans leur croissance et leur développement; la mort est l'évolution normale pour les parasites obligatoires. En situation de rencontre, le parasite exploite les ressources nutritionnelles de l'hôte. L'organisme exploiteur est avantagé dans l'association, où il exprime toutes ses potentialités biologiques de croissance et de développement, alors que l'organisme exploité, l'hôte, est défavorisé. Le parasitisme se distingue de la concurrence par la situation qui préexiste à l'état isolé pour chaque organisme. Toutefois, au niveau des éléments du groupe, les effets mesurés ne se distinguent pas des effets mesurés dans certains cas de concurrence : la croissance et le développement de chaque organisme subissent des actions antagonistes liées à la présence de l'autre organisme, l'une favorable et l'autre défavorable. La même confusion existe au même niveau entre la nature des effets de coopération ou de neutralisme et le processus biologique de la symbiose, où les actions sur la croissance sont en synergie.

II apparaît donc que pour une plante cultivée $A$, désavantagée dans la concurrence, et une mauvaise herbe $B$, qui peut être soit avantagée, soit désavantagée, les effets mesurés peuvent être aussi bien dus à des effets de rencontre dans la même cellule de ressources, qu'à des processus biologiques. Les écologistes parleront plus volontiers de processus d'exploitation, reprenant ainsi un terme utilisé antérieurement dans un sens plus restrictif (Lemee, 1978) : exploitation nutritive pour un facteur limitant du milieu (compétition), chimique (allélopathie) ou parasitaire (parasitisme occasionnel). En pathologie, les processus qui conduisent à un antagonisme entre microorganismes regroupent de façon analogue des phénomènes d'antibiose, d'hyperparasitisme et de compétition nutritive 
(Alabouvette et al. 1983; Sill, 1982). Toutefois, la notion d'antagonisme a tendance à prendre actuellement un sens plus large que la seule inhibition directe de l'agent pathogène par un germe ou une population de son environnement, en considérant les actions (directes et indirectes) exercées par les microorganismes et limitant l'expression de la maladie (Olivier, 1983).

Il est théoriquement possible, dans une culture annuelle d'attribuer un certain pourcentage de perte de rendement à chacun des processus d'exploitation dus aux mauvaises herbes. En fait, la nuisibilité adventice directe, mesurée sur la plante cultivée, ne peut qu'être reliée aux phénomènes de concurrence dans leur ensemble. D'un point de vue pratique, en effet, des valeurs expérimentales de seuils de nuisibilité sont recherchées pour chaque espèce adventice dans des cultures différentes (Zimdahl, 1980; Glauninger \& Holzner, 1982). Pour cette raison, il ne sera envisagé dans la suite de l'article que des situations de concurrence bispécifique pour raisonner les seuils de nuisibilité adventice sous l'aspect de la perte de production.

\section{Seuils de nuisibilité due aux mauvaises herbes : le seuil biologique de nuisibilité}

\section{Seuils de nuisibilité adventice}

La notion de seuil de nuisibilité est liée au type de nuisibilité adventice que l'on redoute principalement (Fig. 2). L'idée simple que le seuil de nuisibilité exprime le niveau d'infestation adventice à partir duquel il est rentable de désherber prête à double confusion. Tout d'abord, la décision de traiter les mauvaises herbes doit être considérée à différents niveaux : celui d'une parcelle cultivée, celui d'une culture de l'assolement, celui d'une exploitation agricole et celui d'une région à caractéristiques socio-économiques définies (Auld et al., 1987). Par ailleurs, déterminer un seuil de nuisibilité pour chacun de ces niveaux exige de faire une synthèse entre des prévisions biologiques (risques d'infestation adventice et espoirs de production potentielle) et des prévisions économiques à plus ou moins long terme : évaluation des coûts de lutte contre les mauvaises herbes et estimation de la valeur des produits récoltés (Cramer, 1967; Cussans et al., 1986).

Sur le plan pratique, il est nécessaire pour mesurer un seuil de nuisibilité de décider quel type de nuisibilité adventice doit être considéré : les effets de concurrence ou les risques d'infestation, les dégâts dus à une mauvaise herbe dominante ou ceux dus à toute la population adventice, la perte de rendement annuelle ou la moyenne sur

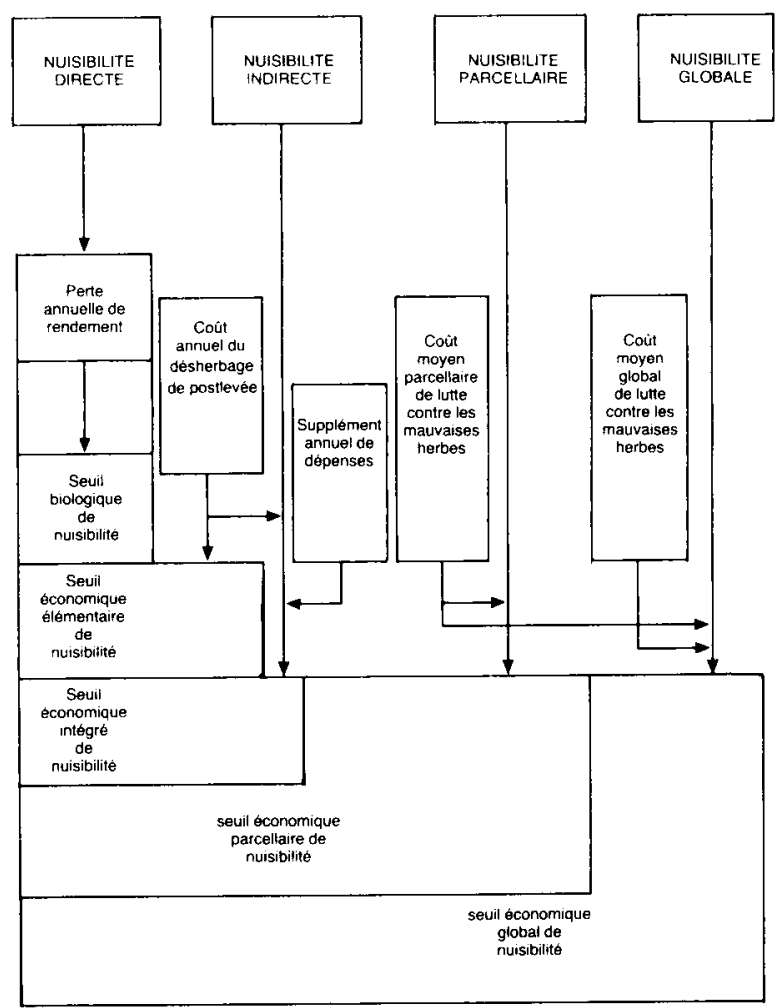

Fig. 2. Seuils de nuisibilité des mauvaises herbes.

plusieurs années ? Plusieurs définitions de seuils de nuisibilité doivent être données pour répondre à des questions aussi diverses que les suivantes, malgré leur ressemblance apparente:

- à partir de quel niveau d'infestation une baisse du rendement de la culture est-elle constatée ?

- à partir de quel niveau d'infestation est-il économiquement nécessaire d'intervenir?

- la destruction complète des mauvaises herbes d'une parcelle cultivée est-elle toujours souhaitable?

- est-il indispensable de faire d'une façon systématique des traitements herbicides?

Sur le plan théorique, la première question met en cause la nuisibilité directe des mauvaises herbes. Comme la relation lie deux paramètres biologiques, la perte de rendement de la plante cultivée et la présence de mauvaises herbes à une période déterminée, le seuil de nuisibilité mesuré est appelé biologique (Koch et Walter, 1983; Cussans et al., 1986). Il est défini comme le niveau d'infestation, à un moment donné, à partir duquel une baisse de rendement de la culture est mesurée. Par exemple, le regroupement des données de 87 essais menés pendant 4 ans dans le sud-ouest de l'Allemagne a montré que des pertes de rendement significatives aux seuils de probabilité de $5 \%$ et $1 \%$ étaient causées respectivement par 22-33 et 27-50 plantes par $\mathrm{m}^{2}$ de vulpin des champs (Alopecurus myosuroïdes 
Huds) dans les cultures de blé d'hiver (Rauber et al., 1980).

Les réponses aux trois autres questions font appel à des éléments économiques chiffrables : ce sont des seuils économiques de nuisibilité qui doivent être définis.

\section{Seuil biologique de nuisibilité}

Relation entre densité adventice et perte de rendement

Souvent défini par le seul paramètre de la densité (Cussans et al. 1986), le seuil biologique de nuisibilité se confond alors avec la densité critique, c'est-à-dire la densité à partir de laquelle une perte de rendement est statistiquement décelable dans des conditions expérimentales définies. Dans des essais où la mauvaise herbe est présente pendant toute la durée de la culture, la recherche d'une densité critique peut être faite selon trois méthodes principales, qui ont fait l'objet de nombreux travaux (Chiarappa, 1971, 1981), et notamment en France ces dernières années :

- le semis de l'adventice à une gamme de densités (soit sur des sols traités en présemis, soit sur des sols préalablement désinfectés) afin d'obtenir des populations artificielles d'une seule mauvaise herbe (Guillemenet, 1972; Wojewedka et Morin, 1983); le repiquage de l'adventice est préféré dans les cultures plantées (Maillet, Abd Elfatah, 1983);

- l'éclaircissage manuel et/ou chimique de peuplements naturels adventices afin d'obtenir une gamme de densités pour une seule espèce adventice (Branthome et al., 1986; Morin et al., 1988);

- l'échantillonnage par placettes dans des parcelles infestées de façon hétérogène par une adventice dominante, de façon à disposer de plusieurs densités pour mesurer la perte de rendement par rapport à un témoin sans mauvaises herbes (Le Clerg, 1971; Guillemenet, 1972; Barralis et Marnotte, 1980; Vacher et Fabre, 1986).

Cependant, en raison des conditions expérimentales et environnementales différentes d'un essai à l'autre, il est rare d'obtenir des densités critiques précises et identiques pour la même adventice. Bien des facteurs font varier les valeurs des densités critiques (Buchanan, 1977; Chisaka, 1977; Koch et al., 1982), qui dépendent en premier lieu des ressources environnementales dont dispose la plante cultivée en l'absence de mauvaises herbes (Rauber, 1977; Poole et Gill, 1987). Connaissant le potentiel de production de la culture, il est tentant de chercher à établir la relation biologique qui lie la densité adventice au rendement ou à la perte de rendement de la plante cultivée, afin de prédire dans une situation donnée les pertes de rendement dues à une mauvaise herbe particulièrement nuisible (Fig. 3). Les modèles qui ont été mis au point sur la base de la méthode des surface de réponse, utilisée en biométrie (Willey et Heath, 1969; Mead et Pike, 1975), ont recherché les fonctions mathématiques qui s'adaptaient le mieux aux données d'expériences de concurrence bispécifique. Diverses fonctions classiques exprimant la diminution du rendement de la plante cultivée lorsque la densité de l'adventice s'élève ont été proposées : polynômes, polynômes inverses, exponentielles, ... (Tableau II). Comme la perte de rendement tend vers une limite asymptotique lorsque la densité s'élève, les modèles basés sur de telles considérations biologiques s'ajustent le mieux aux données lorsque la gamme de densités est très étendue. Parmi les plus appropriés se rencontrent l'hyperbole rectangulaire (Cousens, 1985a), certaines fonctions polynomiales
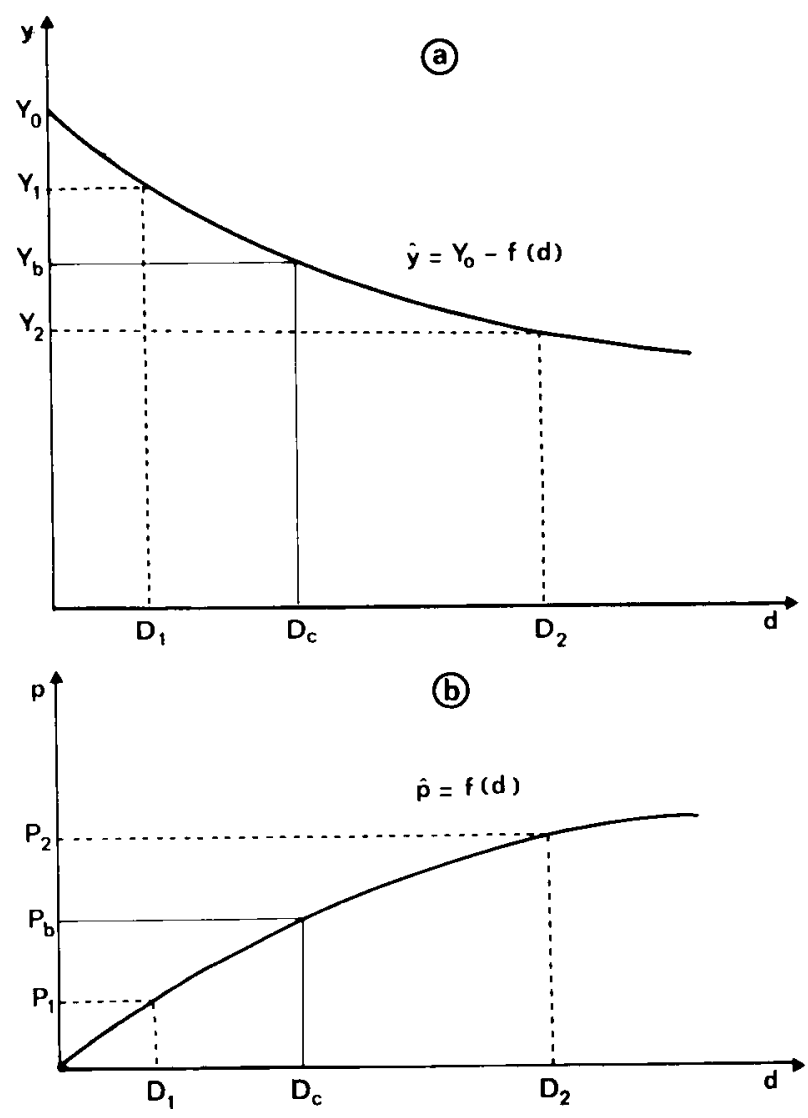

Fig. 3. Seuil biologique de nuisibilité : influence de la densité adventice (d), a. sur le rendement (y) de la plante cultivée, b. sur la perte de rendement $(p)$ de la plante cultivée.

Soient $\hat{y}=Y_{0}-f(d)$ et $\hat{p}=f(d)$ les relations biologiques qui lient respectivement le rendement $y$ et la perte de rendement $p$ à la densité adventice $d, Y_{0}$ étant le rendement du témoin sans adventice.

A deux densités quelconques $D_{1}$ et $D_{2}$ correspondent soit les rendements respectifs $Y_{1}$ et $Y_{2}$, soit les pertes de rendement respectives $P_{1}$ et $P_{2}$. A la densité critique $D_{c}$, le rendement $Y_{b}$ est significativement inférieur (au seuil de probabilité choisi) au rendement du témoin $Y_{0}$; la perte de rendement correspondante est $P_{b}$. 
Tableau II. Exemples de relations utilisées en concurrence bispécifique entre la densité d'une adventice (d) et le rendement d'une plante cultivée (y) (d'après Cousens, 1985a; Cousens et al., 1987).

1. Relations fondées sur des considérations biologiques.

(densité de l'adventice variant de 0 à $+\infty$

limite asymptotique égale à $O$ ou $A$ )

Fonction

$\hat{y}=Y_{0}(1+a d)^{-1}$

$\hat{y}=Y_{0}\left(1-\frac{a d}{a d+b}\right)$

$\hat{y}=Y_{0}(1+a d)^{-b}$

Hyperbole rectangulaire

$\hat{y}=Y_{0}\left(1-\frac{a d}{1+b d}\right)$

$\hat{y}=Y_{0} b e^{-a d}$

$\hat{y}=Y_{0}\left(1-b e^{-a d}\right)$

$\hat{y}=Y_{0}-b\left(1-e^{-a d 2}\right)$

II. Relations empiriques simples

(gamme de densités adventices faibles

limite tendant vers $+\infty$ )

Equation linéaire

$\hat{y}=Y_{0}-a d$

Madeira et al., 1984

Equation quadratique

$\hat{y}=Y_{0}-a d+b d^{2}$

Schweizer et Bridge, 1982

Equations avec terme de puissance 1/2

$\hat{y}=Y_{0}-a \sqrt{d}$

Dew, 1972

$\hat{y}=Y_{0}-a \sqrt{d}+b d$
Référence bibliographique

Chisaka, 1977

Hakansson, 1983

Watkinson, 1981

Cousens et al., 1985

Medd et al., 1985

Wilson et Cussans, 1983

Williams et Hayes, 1984

$Y_{0}$ : rendement témoin de la plante cultivée en l'absence de l'adventice. $a, b, c$ : termes constants.

(Watkinson, 1981; Hakansson, 1983) et des fonctions exponentielles (Wilson et Cussans, 1983).

Pour une gamme de densités adventices faibles correspondant le plus souvent aux infestations de cultures à haute productivité, une équation linéaire ou quadratique suffit généralement à décrire la relation (Fig. 4). Parfois, la relation qui lie la perte de rendement et la densité est exprimée par rapport à la racine carrée de la densité adventice (Wiese, 1971; Dew, 1972; O'Sullivan et al., 1982).

Malgré leur manque de signification biologique lorsque la densité de l'adventice excède la gamme de densités expérimentées, ces relations empiriques permettent de préciser les densités critiques d'espèces adventices nécessitant une opération de désherbage dans une culture. Elles peuvent aider également à l'interprétation des effets de concurrence sur les composantes du rendement (Covarelli et Tei, 1984; Caussanel et al., 1988).

Afin d'améliorer l'ajustement aux résultats expérimentaux, la densité de la plante cultivée a été introduite dans le modèle. Des modèles de régression multiple du rendement de la plante cultivée en fonction de sa propre densité et de celle de la mauvaise herbe ont été établis par 

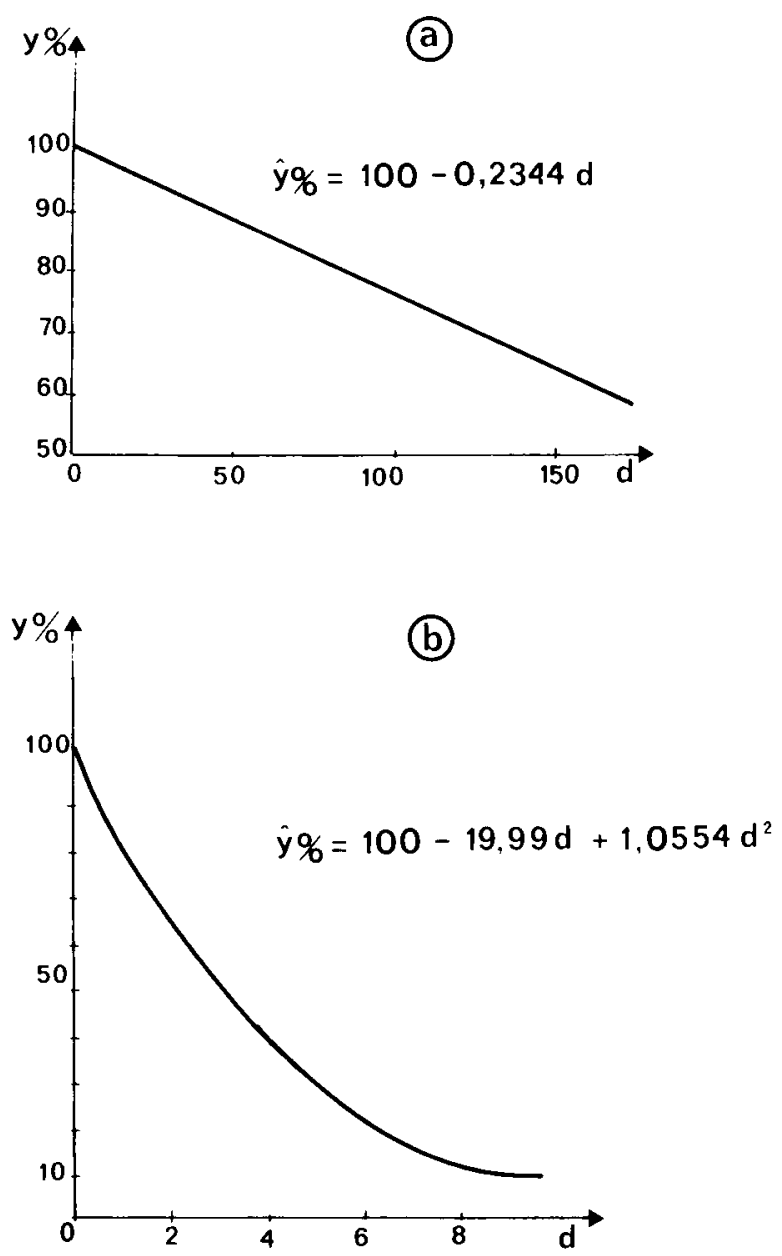

Fig. 4. Exemples de relations entre le rendement d'une culture et la densité d'une adventice en situation de concurrence bispécifique (d'après Caussanel et al., 1986). a. Diminution du rendement du blé d'hiver en présence de ray-grass d'italie (Lolium multiflorum Lam). b. Diminution du rendement de la tomate de semis en présence de morelle noire (Solanum nigrum $\mathrm{L}$.) . $y \%=$ pourcentage de rendement obtenu en présence de l'adventice par rapport au rendement du témoin (= 100). $d=$ nombre de plantes par $m^{2}$.

exemple pour l'orge en présence de Sinapis alba L. (Hakansson, 1983; Cousens, 1985b) ou pour le blé soumis à la concurrence d'Avena fatua L. (Carlson et Hill, 1985), de Lolium rigidum Gaud. (Medd et al., 1985) ou d'Agrostemma githago L. (Watkinson, 1981).

\section{Structures de la population adventice et pertes de rendement}

En fait, comme le seuil biologique de nuisibilité est défini par rapport à la nuisibilité directe des mauvaises herbes, la densité n'est qu'un des éléments à retenir pour déterminer un tel seuil de nuisibilité. Adventice ou autre, toute population peut être considérée comme un (ou plusieurs) ensemble(s) d'individus de la même espèce pouvant vivre en commun à un moment de leur cycle de développement et interagir alors sur les mécanismes de leur reproduction; chaque population présente plusieurs types de structure (sociale, spatiale, temporelle, génétique, ..) et plusieurs niveaux de structuration (Legay et Debouzie, 1985). Aussi, les relations entre plantes cultivées et adventices doivent être abordées sous leurs aspects démographiques, génétiques et "fonctionnels", ces derniers étant relatifs au fonctionnement intégré des plantes selon leurs réponses physiologiques de croissance et de développement aux conditions d'offre environnementale qui leur sont fournies.

Parmi les aspects démographiques, cinq catégories principales d'effets (Fig. 5) peuvent être distinguées (Jacquard, 1980) :

- la densité (effets de densité) de la plante cultivée et de chaque mauvaise herbe;

- l'espèce pour chaque mauvaise herbe et le cultivar pour la plante cultivée (effet partenaire);

- les proportions des espèces entre elles, soit entre la plante cultivée et les mauvaises herbes, soit entre les mauvaises herbes elles-mêmes (effet proportions);

- la répartition sur le terrain, qu'il s'agisse de l'écartement sur le rang et entre les rangs pour la plante cultivée ou de la distribution des levées de mauvaises herbes sur le terrain (effets de structure spatiale);

- la période de concurrence interspécifique entre la plante cultivée et les mauvaises herbes mais aussi entre les mauvaises herbes elles-mêmes (effets de structure temporelle).
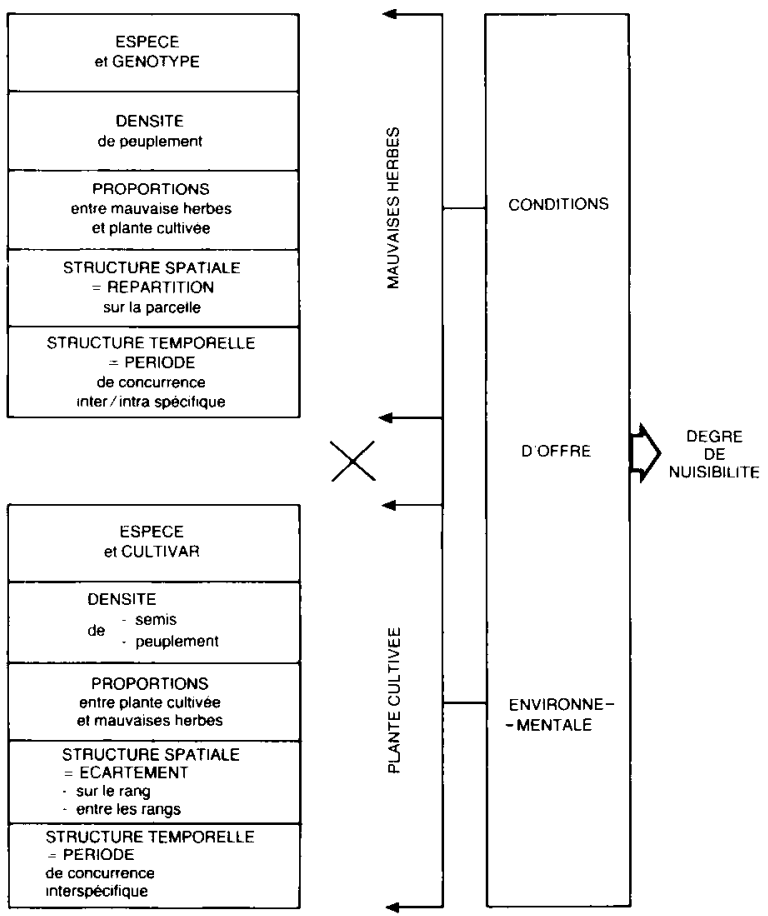

Fig. 5. Facteurs modifiant le degré de nuisibilité des mauvaises herbes dans une culture. 
Comme la levée de chaque adventice est déterminée par la conjonction de plusieurs propriétés de la semence et du milieu ambiant (Montegut, 1975; Egley, 1986), les effets de structure temporelle déterminent la connaissance de la période critique des mauvaises herbes. Définie comme la période pendant laquelle la présence d'adventices entraîne une perte de rendement mesurable, la période critique indique la meilleure période d'intervention pour la réalisation d'un ou de plusieurs traitements herbicides. Sa détermination précise exige une méthodologie adéquate (Nieto et al., 1968; Peters, 1972; Weaver, 1984; Dawson, 1986). La méthode consiste à utiliser les résultats de deux expériences complémentaires afin de tracer deux courbes de diminution du rendement en fonction de la durée de concurrence.

- la première expérience consiste à laisser se développer les mauvaises herbes pendant des périodes de plus en plus longues avant de les éliminer, mécaniquement ou chimiquement (Fig. 6a); la concurrence précoce est ainsi mesurée pour différentes périodes depuis la levée et un seuil de concurrence précoce Sp est établi;

- la seconde expérience a pour objectif de maintenir propre la culture depuis la levée jusqu'à des dates de plus en plus reculées dans le temps (Fig. 6b) : un seuil de concurrence tardive (St) est alors déterminé.

En reportant les deux courbes sur le même graphique, la période critique $T_{c}$ apparaît entre Sp et St (Fig. 6c).

Dans les associations entre plantes cultivées et mauvaises herbes, les études de concurrence se limitent généralement à la prise en considération des seuls aspects démographiques. C'est ainsi que la perte de rendement a été analysée en fonction à la fois de la densité et de la période de concurrence d'une mauvaise herbe par la méthode de la régression multiple dans une culture de blé ou d'orge (O'Donovan et al., 1985) ou par la méthode des surfaces de réponse dans une culture de soja (Keisling et al., 1984).

Pourtant, l'approfondissement de nos connaissances sur la biologie des mauvaises herbes met de plus en plus en relief l'intérêt des aspects

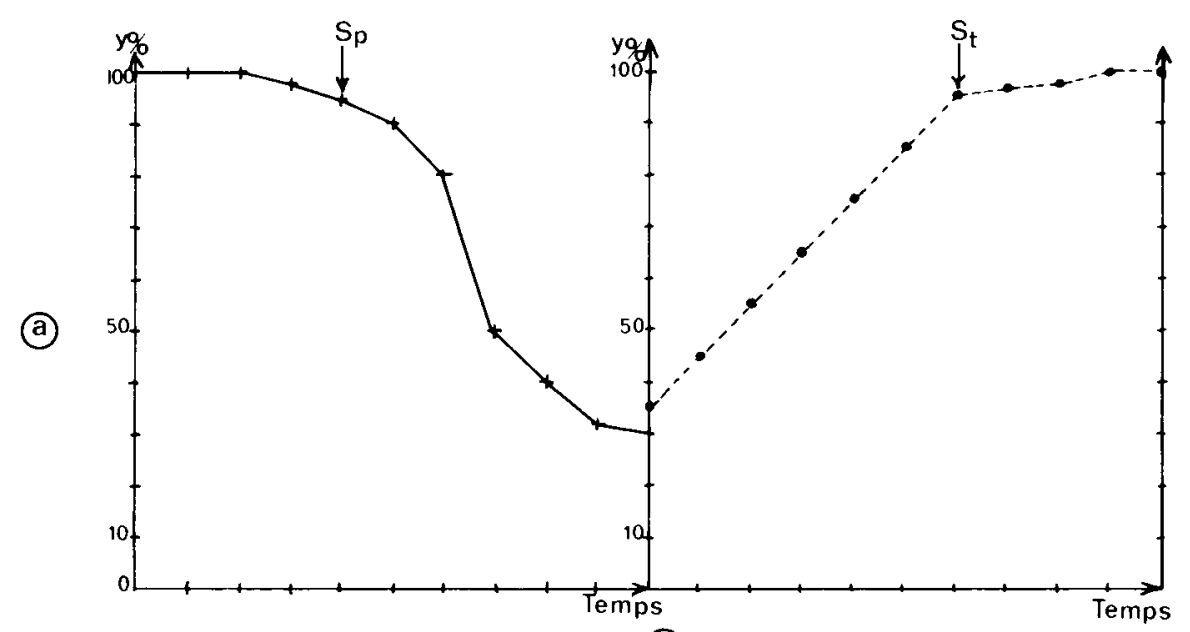

(b)

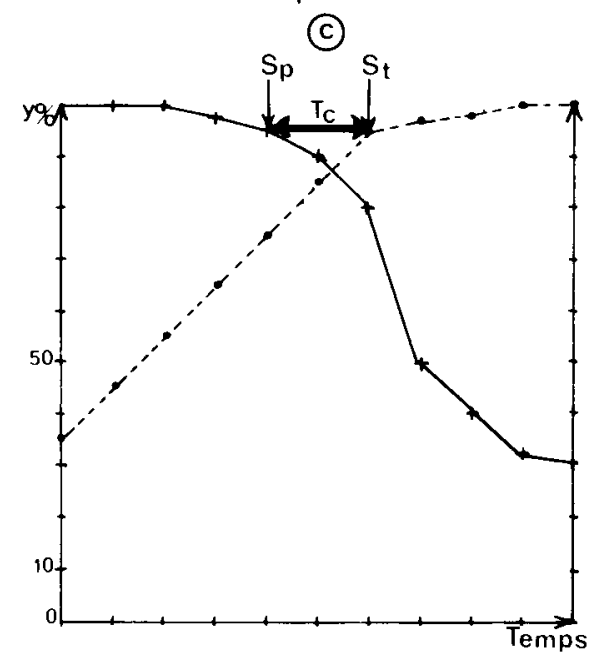

Fig. 6. Détermination de la période critique (d'après Nieto et al., 1968 et Dawson, 1986). a) Relation entre le rendement (en \%) et la durée d'enherbement (exprimée en temps). Sp = seuil de concurrence précoce. b) Relation entre le rendement (en \%) et la durée de désherbage (exprimée en temps). St = seuil de concurrence tardive. c) Relations entre le rendement (en \%) et la période après la levée exprimée en temps. Tc $=$ période critique. 
génétiques, en raison de l'existence d'une variabilité infraspécifique dans les populations de mauvaises herbes, entraînant un polymorphisme de la résistance aux herbicides (Harlan, 1982; Gasquez, 1984; Darmency, 1987; Gressel, 1987). Au niveau des plantes cultivées comme à celui des plantes adventices, les relations de concurrence s'en trouvent modifiées (Mondragon, 1988) et le raisonnement des seuils de nuisibilité dans la pratique du désherbage ne peut sous-estimer les risques de réinfestation par des populations adventices résistantes à certains herbicides (Ammon, 1988). En intégrant le fonctionnement du développement et de la croissance végétale des espèces en concurrence (aspects fonctionnels), les modèles de simulation utilisent des paramètres appropriés à l'écosystème décrit (Spitters et Van Den Bergh, 1982; Spitters et Aerts, 1983; Kropft, 1988).

Dans les dispositifs expérimentaux de plein champ (Fig. 7), I'utilisation d'une plante cultivée comme adventice facilite l'analyse expérimentale des effets de concurrence bispécifique, lorsque sont pris en considération les divers niveaux de structure de l'espèce "adventice" et les techniques culturales de production de l'espèce cultivée (Caussanel et al., 1988). L'expérimentation sur une ou plusieurs adventices, plus proche de la réalité agronomique, accroît cependant la complexité des niveaux d'étude (Haizel et Harper, 1973; Hakansson, 1986; Abd Elfatah, 1987). En plein champ, l'interprétation des résultats exigerait une analyse des structures spatiotemporelles des peuplements végétaux présents, d'autant plus que les relations de concurrence sont modifiées par les techniques culturales (Sagar,

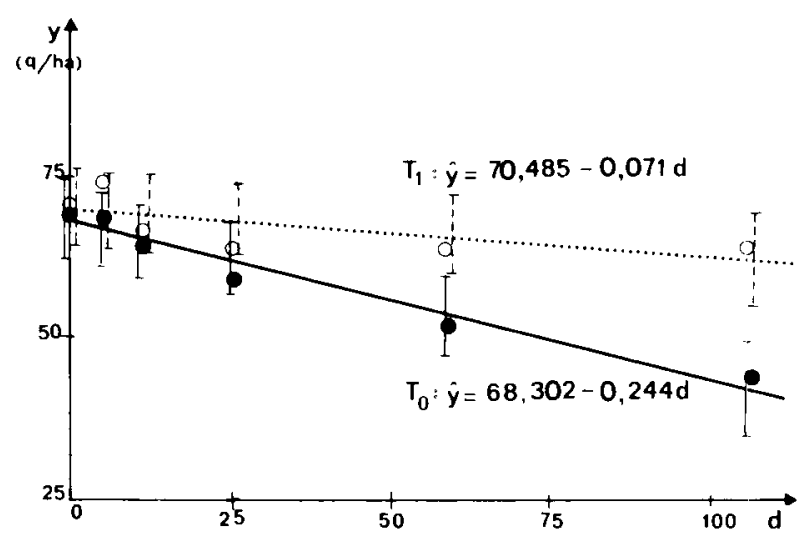

Fig. 7. Relation entre la densité réelle moyenne de population (d) d'une avoine de printemps et le rendement d'un blé de printemps $(y)$ en fonction du traitement herbicide au diclofopméthyl (d'après Caussanel et al., 1988). $T_{0}=$ aucun traitement au diclofométhyl $(\bullet)$ intervalle de confiance. $T_{1}=$ traitement au diclofopméthyl au début du tallage (o) intervalle de confiance I.
1968; Reeves, 1976; Andersson, 1986) et notamment par les traitements herbicides (Eggers, 1975). Des méthodes descriptives utilisées en biologie des populations (Chessel, 1978; Thioulouse, 1985, 1987) ont été récemment appli-quées aux populations de mauvaises herbes de cultures de maiis (Kheddam, 1988).

\section{Seuils de nuisibilité adventice : les seuils économiques de nuisibilité}

\section{Seuil économique annuel de nuisibilité}

Sur une base annuelle de données, le seuil économique annuel de nuisibilité tient compte du coût des opérations de désherbage de postlevée mais aussi, éventuellement, des dépenses supplémentaires engagées pour supprimer la nuisibilité indirecte des mauvaises herbes. II représente le niveau d'infestation (atteint au moment conseillé pour éliminer les mauvaises herbes) à partir duquel une opération de désherbage devient rentable, compte tenu du prix de revient de cette opération et de la valeur de la récolte. $\mathrm{Si}$ la valeur du produit récolté est appréciée sous son seul aspect quantitatif, c'est le seuil économique élémentaire de nuisibilité qui est défini. II dépend de la relation qui lie le niveau d'infestation adventice et la perte de rendement, de la valeur ajouté au produit récolté résultant de l'élimination des mauvaises herbes et du coût de l'opération de désherbage (Garburg et Heitefuss, 1975). Si les charges supplémentaires occasionnées par l'élévation du coût du produit commercialisable due à la nuisibilité adventice indirecte sont inclues dans le prix de revient de la lutte contre les mauvaises herbes, ce seuil économique annuel est dit intégré (Fig. 8a). En termes économiques, il s'agit du seuil au-delà duquel l'accroissement de la dépense nécessaire pour lutter contre les mauvaises herbes n'est plus compensé par l'accroissement du prix de vente du produit commercialisé. L'ampleur de l'infestation adventice au moment du désherbage et la réussite du traitement herbicide modifient les valeurs des seuils de nuisibilité (Fig. $8 \mathrm{~b}$ et $\mathrm{C}$ ). A titre d'exemple, le seuil économique annuel de nuisibilité de la folle avoine (Avena fatua $L$.) dans le blé a été calculé dans les conditions de l'agriculture australienne et s'élève à 7 plantes d'avoine par $\mathrm{m}^{2}$ (Auld et Tisdell, 1985). Selon le même mode de calcul dans les conditions des cultures de soja de Caroline du Nord, le seuil économique annuel de nuisibilité de l'amaranthe hybride (Amaranthus hybridus L.) serait de 2,9 plantes pour $10 \mathrm{~m}$ de longueur de rang (Marra et Carlson, 1983). 

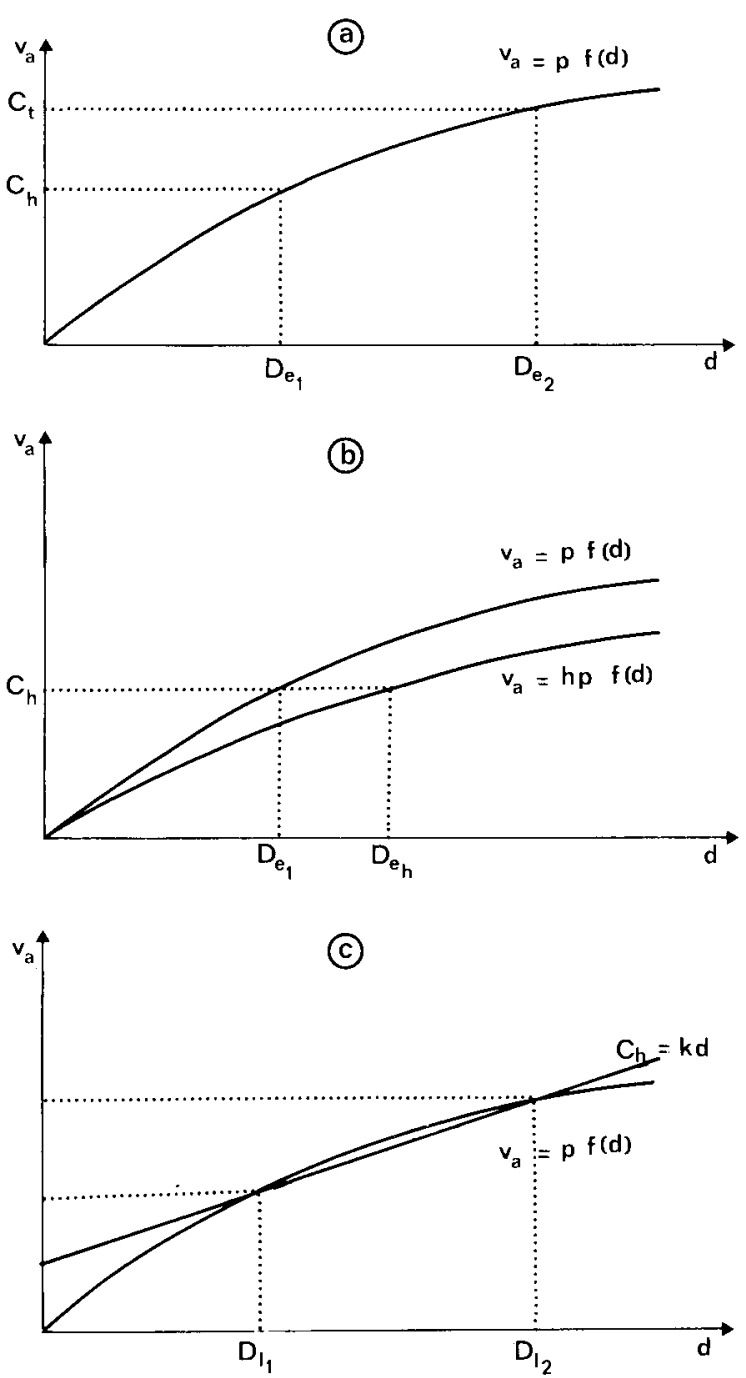

Fig. 8. Seuils économiques annuels de nuisibilité (d'après Auld et al., 1987). a. Soit va $=\mathrm{p} f(d)$ la relation économique qui lie la valeur ajoutée au produit récolté résultant de l'élimination des mauvaises herbes (va) et la densité adventice (d), $p$ étant le prix de vente de l'unité du produit récolté. Le coût du traitement est supposé indépendant de la densité adventice et le traitement efficace à 100 p. 100 . Si le coût de l'opération de désherbage $\left(C_{h}\right)$ est uniquement pris en considération, le seuil économique élémentaire de nuisibilité est atteint pour une densité $\mathrm{D}_{\mathrm{e} 1}$. Si le coût de la lutte contre les mauvaises herbes $\left(C_{t}\right)$ comprend les charges supplémentaires dues à la nuisibilité indirecte des mauvaises herbes, le seuil de nuisibilité économique intégré est obtenu pour une densité $D_{\mathrm{e} 2}$ plus élevée que la précédente. $b$. En ne considérant que le coût de l'opération de désherbage et au cas où le désherbage n'est efficace qu'à h p. 100, l'équation précédente devient va $=h p f(d)$. Le seuil économique de nuisibilité se déplace vers une densité plus élevée que $D_{e 1}: D_{e h}$. c. Si le coût de l'opération de désherbage n'est pas indépendant de la densité et qu'il s'élève linéairement par exemple avec la densité adventice $\left(C_{n}=k d\right)$, deux densités critiques existent, $\mathrm{Dl}_{1}$ et $\mathrm{Dl}_{2}$, en dessous et au-dessus desquelles respectivement le désherbage est trop onéreux pour l'efficacité escomptée.

\section{Seuil économique pluriannuel de nuisibilité}

Calculé sur plusieurs années, le seuil économique pluriannuel de nuisibilité fait intervenir les risques de nuisibilité potentielle des mauvaises herbes, dus à la flore potentielle de la parcelle cultivée (seuil économique parcellaire de nuisibilité) et de l'exploitation (seuil économique global de nuisibilité). Au niveau de la parcelle comme au niveau de l'exploitation agricole, il existe plusieurs façons de l'appréhender : une optique prévisionnelle (Walker, 1983), une optique d'optimum économique (Cousens, 1986) et une optique de sécurité économique.

II devient difficile sur plusieurs années de raisonner le désherbage en fonction de la seule densité adventice, alors que la principale crainte est de voir le niveau d'infestation adventice s'accentuer avec le temps. Afin de décider une opération de désherbage dans la pratique de la lutte phytosanitaire, les spécialistes de la Protection des Végétaux préfèrent utiliser les concepts de "seuils de tolérance» (Baggiolini, 1977), de "seuils d'intervention" (Walker, 1983) ou de "seuils de nuisibilité techniques» (Tissut et Severin, 1984). II s'agit ici d'un seuil auquel la décision de traiter doit être prise, avant que le seuil intégré de nuisibilité soit dépassé. Le but est d'intervenir sur une densité de population adventice au moment où elle atteint un seuil prédit. Comme un grand nombre de facteurs sont susceptibles de faire varier ce seuil (Chiang, 1979), le seuil prédit d'intervention est généralement obtenu, soit en l'établissant à partir d'une base de données expérimentales pluriannuelles, soit en adaptant à la situation locale considérée une valeur utilisée régionalement, soit encore en calculant le bénéfice économique escompté pour le niveau d'infestation paraissant nécessiter l'intervention. Plusieurs seuils d'intervention ont été calculés en entomologie, en nématologie ou en phytopathologie (Walker, 1983). En biologie des populations de mauvaises herbes, les corrélations entre le nombre de semences dispersées puis enfouies dans le sol et le nombre de plantules levées les années suivantes pourraient être calculées. Elles sont à établir pour chaque espèce adventice en raison de la diversité des types de dormance chez la plupart des semences de mauvaises herbes (Roberts, 1972; Chadoeuf-Hannel, 1985). Des corrélations significatives ont été obtenues pour le vulpin des champs (Alopecurus myosuroïdes Huds) entre le nombre de semences levées en serre dans des échantillons de sol et les densités d'infestation correspondantes au champ d'où provenait l'échantillon, au cours de l'automne ou du printemps suivant (Naylor, 1970). En plein champ, le taux annuel moyen de levée d'espèces adventices fréquentes est difficile à corréler avec le nombre de semences apparemment viables par parcelle, en raison notamment des conditions climatiques de l'hiver ou de la profondeur d'enfouissement des semences 
(Barralis et Chadoeuf, 1988). Des modèles décrivant l'évolution quantitative de la flore adventice sur plusieurs années ont déjà été envisagés (Mortimer, 1983; Wilson et al., 1984; Debaeke, 1988). Jusqu'ici, les valeurs prédites par modélisation de populations d'Avena fatua $L$. (Maniove et al., 1982) ou de Bromus sterilis L. (Firbank et al., 1985) à partir des stocks semenciers de l'année précédente ont montré de fortes différences par rapport aux valeurs observées selon les conditions climatiques de l'année ou les techniques culturales employées.

Une approche plus analytique des variations des populations de mauvaises herbes dans le milieu cultural conduit au calcul de seuils économiques optimaux de nuisibilité d'adventices particulièrement redoutables. Le seuil économique optimal de nuisibilité (Cousens, 1986) est défini comme le niveau d'infestation à partir duquel les mauvaises herbes devraient être désherbées afin d'optimiser les retombées financières consécutives aux opérations intégrées de désherbage. L'accent est mis sur la modélisation du cycle biologique de la mauvaise herbe entreprise depuis plusieurs années dans certains travaux de recherche (Sagar, 1970; Rauber et Koch, 1975; Sagar et Mortimer, 1976; Watkinson, 1981; Cussans et Moss, 1982; Mortimer et Putwain, 1984; Aarts, 1986; Zwerger et Hurle, 1988). Le modèle complet utilise des paramètres biologiques qui ont été déterminés dans ces travaux antérieurs et ceux qui lient le rendement à la densité adventice, ainsi que des paramètres économiques faisant intervenir le coût de l'application herbicide et le prix du produit récolté sous certaines conditions de qualité (pourcentage de contamination par les semences de mauvaises herbes par exemple). Comme le désherbage de l'espèce adventice réduit son abondance les années suivantes, le seuil économique annuel de nuisibilité tend à décroître sous la pression d'un désherbage rationnel, après un certain nombre d'années dépendant du potentiel semencier du sol à l'origine (Fig. 9). En conditions de monoculture du blé d'hiver, le modèle prévoit que le bénéfice économique optimal à long terme sur dix années est obtenu pour une densité de 2 à 3 plantules par $\mathrm{m}^{2}$ de folle avoine (Avena fatua $L$.) dans le cas d'un seul traitement annuel au difenzoquat (Cousens et al., 1986) et de 7,5 plantes par $\mathrm{m}^{2}$ de vulpin des champs (Alopecurus myosuroïdes Huds) dans le cas d'un seul traitement annuel au chlortoluron (Doyle et al., 1986).

Bien des incertitudes demeurent néanmoins en ce qui concerne certains paramètres comme le niveau probable d'infestation adventice après le traitement (nouvelles levées de l'adventice traitée ou développement d'autres adventices), l'effi-

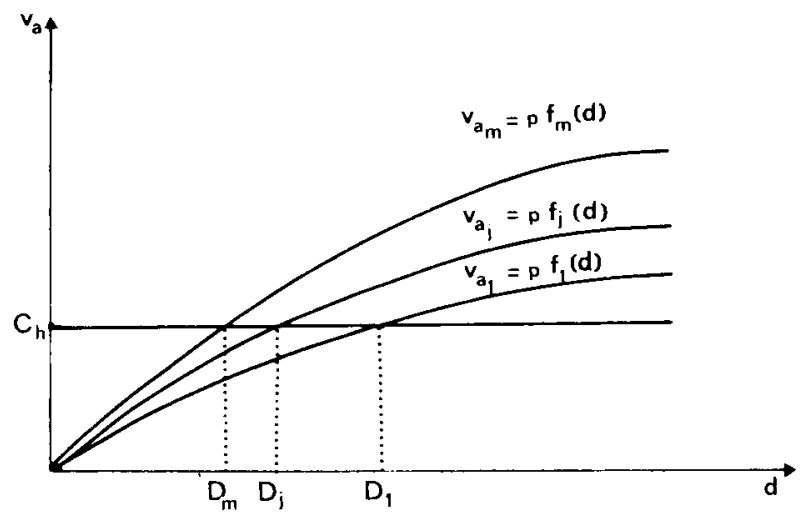

Fig. 9. Seuils économique pluriannuels de nuisibilité : «seuil optimal” (d'après Cousens et al., 1986 et Auld et al., 1987). Soit va $a_{1}=p f_{1}$ (d) la relation économique qui lie pour l'année 1 la valeur ajoutée au produit récolté résultant de l'élimination des mauvaises herbes (va) et la densité adventice $d$. Soit $D_{1}$ le seuil économique annuel de nuisibilité pour l'année 1 . Lorsque la pression de désherbage réduit la densité adventice après $\mathrm{j}$ années, la valeur ajoutée a augmenté en fonction de la densité et le seuil de nuisibilité pour l'année j est obtenu pour une densité $D_{1}$ inférieure à $D_{1}$. Le seuil optimal $D_{m}$ atteint après $\mathrm{m}$ années est donc inférieur au seuil économique annuel de départ.

cacité du traitement herbicide (rendue partielle en fonction des conditions environnementales) ou l'évolution des prix et des coûts sur une longue durée (coûts de désherbage, prix de vente du produit récolté). La relation biologique est elle-même sujette à variations : pour une densité de 20 plantes par $\mathrm{m}^{2} \mathrm{~d}^{\prime}$ Echinochloa crus galli $L$., levées après le repiquage du riz, les pertes de rendement ont varié entre $8 \%$ et $17 \%$ (Chisaka, 1977). Aussi, comme aucun des seuils pluriannuels définis précédemment ne prévoit d'intervalles de confiance aux valeurs numériques utilisées pour certains paramètres dans la modélisation, le concept de seuil économique de sécurité a été introduit. Comme le montrent les résultats des déterminations de seuils optimaux de nuisibilité (Tableau IIt), il existe une limite inférieure et une limite supérieure pour chaque valeur trouvée dans différentes situations de techniques culturales. Les relations économiques entre la valeur ajoutée au produit récolté résultant de l'élimination des mauvaises herbes et le niveau d'infestation adventice doivent se présenter, pour une utilisation pratique, sous la forme d'une surface de réponse, dont les limites supérieure et inférieure correspondent à des cas extrêmes (Fig. 10). Actuellement, la généralisation à partir de résultats expérimentaux a conduit à la recommandation de seuils de nuisibilité aidant à la décision dans le désherbage de certaines adventices (Tableau IV). L'utilisation de ces valeurs moyennes requiert une adaptation locale ou régionale, d'autant plus que les seuils de nuisibilité recommandés pour décider de l'op- 
Tableau III. Seuils de nuisibilité économiques optimaux de la folle avoine (Avena fatua L.) avec marges de sécurité dans le blé d'hiver (exprimés en nombre de plantes par m²) (d'après Cousens et al., 1986).

\begin{tabular}{llll} 
Technique culturale & $\begin{array}{l}\text { Seuil de nuisibilité } \\
\text { économique optimal }\end{array}$ \\
\hline + Travail superficiel & & & \\
- pailles brûlées & 2,2 & - & 3,3 \\
$\quad$ - pailles non brûlées & 2,3 & - & 3,2 \\
+ Labour & & & \\
- pailles brûlées & 2,4 & - & 2,5 \\
- pailles non brûlées & 2,0 & - & 2,4 \\
\hline
\end{tabular}

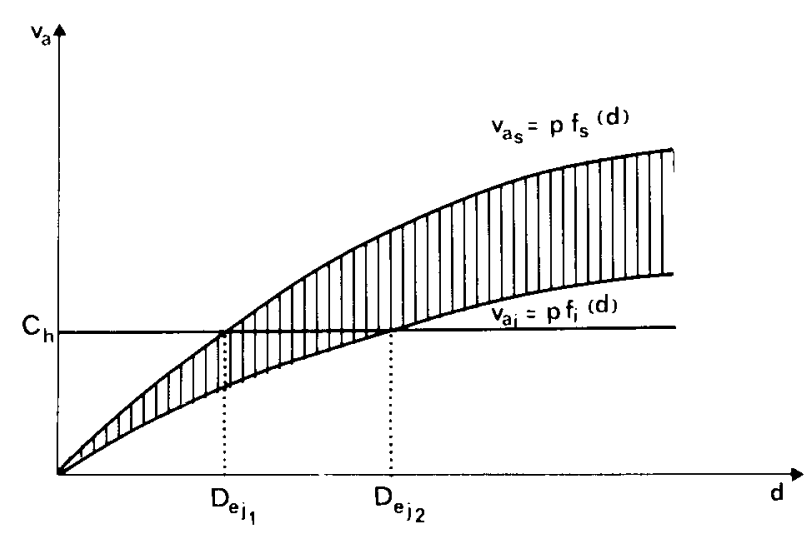

Fig. 10. Seuils pluriannuels économiques de nuisibilité : seuil de sécurité (d'après Auld et al. 1987). La relation va $=p \mathrm{f}(\mathrm{d})$ présentée dans la figure 9 est calculée avec deux limites de sécurité, l'une inférieure $v a_{\mathrm{i}}$, l'autre supérieure $v \mathrm{a}_{\mathrm{s}}$. Entre deux seuils économiques extrêmes de nuisibilité qui sont respectivement $D_{e j 1}$ et $D_{e j 2}$, existe une gamme de seuils économiques de nuisibilité, où la valeur ajoutée au produit récolté n'est pas déterminée avec précision.

portunité d'un traitement désherbant peuvent être déterminés à partir de différents critères (Heitefuss et al., 1987; Marshall, 1987).

La lutte contre le vulpin des champs (Alopecurus myosuroïdes Huds), conduite sur ces principes dans une rotation culturale, a permis, dans une étude expérimentale portant sur 20 parcelles en Basse-Saxe, de réduire les surfaces désherbées chimiquement de $100 \%$ en 1980 à $35 \%$ en 1985 (Niemann, 1986). En Basse-Saxe également, les coûts de désherbage ont été réduits dans l'orge d'hiver en appliquant les recommandations basées sur l'utilisation de seuils économiques de nuisibilité (Wahmhoff et Heitefuss, 1984). Des études similaires, entreprises pour lutter contre des graminées (Apera spica venti (L.) P. Beauv.) ou des dicotylédones (Viola arvensis L.) dominantes dans le seigle, n'ont montré aucun effet préjudiciable sur le rendement, la contamination du grain en semences ou l'humidité du grain (Beer, 1986). Toutefois, cer- taines espèces comme Alopecurus myosuroïdes Huds. tallent très différemment d'un sol à un autre ou d'une année à une autre, ce qui peut être source d'erreurs dans certaines décisions de désherbage fondées sur le nombre de plantes par $\mathrm{m}^{2}$ (Kemmer et Hurle, 1984). Aussi, les stades atteints par la plante cultivée et par la mauvaise herbe au moment du désherbage ont été introduits dans ces modèles prévisionnels ainsi que diverses marges de sécurité (Gerowitt, 1987). L'analyse du potentiel semencier du sol, dans des situations expérimentales où le désherbage était conduit en fonction de seuils préconisés de nuisibilité, montre peu de différences avec des situations de désherbage intensif après piusieurs années dans une rotation culturale définie (Hagemeister et Heitefuss, 1986; Kees, 1986).

Avec l'utilisation accrue des ordinateurs, le développement de ces modèles tend à inclure des paramètres de plus en plus nombreux pour les rendre plus crédibles auprès des agriculteurs qui continuent de traiter sur la base d'appréciations subjectives (Tait, 1977; Mumford, 1982). Les essais de validité en plein champ indiquent que ces modèles sont de plus en plus fiables, même si de nombreuses données expérimentales sont encore à recueillir pour la plupart des espèces. Les situations de concurrence plurispécifique sont encore peu abordées en modélisation, de même que l'influence des rotatisins culturales, connues pour modifier la concurrence des mauvaises herbes dans les céréales (Sebillotte, 1967).

Des tentatives ont été faites pour déterminer les seuils biologiques de nuisibilité d'une flore plurispécifique, soit par la relation entre la densité totale et le rendement (Eggers et Niemann, 1980; Jensen, 1985), soit par la liaison entre le recouvrement adventice et le rendement (Kolbe, 1977; Bleiholder et Nuyken, 1986), soit par l'emploi de valeurs traduisant une équivalence de poids de matière sèche entre la plante cultivée et chaque espèce de mauvaise herbe (Wilson, 1986).

Dans une optique plus phytosanitaire, il a été proposé, pour décider de l'opportunité d'un traitement de postlevée, de se référer à des valeurs prédictives : la perte de rendement, déterminée expérimentalement dans une série d'essais herbicides, est exprimée en fonction de la densité adventice et du meilleur rendement parcellaire de chaque essai. De telles régressions multiples ont été proposées pour la folle avoine dans les céréales (Loubaresse et al., 1975) ou pour les mauvaises herbes du colza (Regnault et Pouzet, 1983). La différence de rendement entre le traité et le témoin (supplément de rendement à espé- 
Tableau IV. Seuils de nuisibilité dans les céréales (exprimés en nombre de plantes par $\mathrm{m}^{2}$ ).

1. Seuils annuels de nuisibilité d'aide à la décision

Neururer, 1975
1976

(1)
Wahmhoff, 1986

(3)

30

20

$-$

Veronica sp.

Vicia villosa Roth

4,3
0,7
9,4
0,7
-
6,1
10,3
9,0
0,8

II. Seuils annuels économiques de nuisibilité

Cousens et: al., 1985

Avena fatua $\mathrm{L}$.

Alopecurus myosuroïdes Huds

III. Seuils pluriannuels économiques de nuisibilité

$8-\quad 12$
$30-50$

Cousens et al., 1985
Avena fatua $\mathrm{L}$. Alopecurus myosuroïdes Huds.
2 à 3

7.5
Aarts et Visser, 1985

(3)

(1) "seuil biologique de nuisibilité" = densité moyenne de mauvaise herbe causant $5 \%$ de perte de rendement à la récolte. (2) "seuil agrotechnique de nuisibilité" = densité moyenne de mauvaise herbe entraînant $5 \%$ de gêne supplémentaire dans la technique de récolte, par rapport au témoin sans mauvaises herbes. (3) "seuil pratique de nuisibilité" = densité moyenne de mauvaise herbe évaluée à l'aide de notations par des essais de pratique agricole entraînant une baisse de rendement (donnée moyenne établie à partir d'un grand nombre de résultats d'essais).

rer) dépend de deux paramètres : le nombre de plantes par $\mathrm{m}^{2}$ du témoin et le meilleur rendement parcellaire de la répétition considérée. Ce type de prévision est aussi utilisé lorsque la perte de rendement est due à deux (Blackshaw, 1986) ou à plusieurs (Pinstrup-Andersen et al., 1976) organismes nuisibles, à condition qu'il n'y ait pas d'interaction entre eux.

\section{Conclusion}

La détermination des seuils de nuisibilité des mauvaises herbes dans les cultures offre, comme il vient d'être vu, des possibilités nouvelles dans le raisonnement de la lutte intégrée en agriculture. Des seuils annuels de nuisibilité utiles à la pratique du désherbage sont déjà d'actualité pour quelques adventices annuelles dans certaines cultures principales annuelles. L'amélioration des modèles prévisionnels devrait permettre dans un proche avenir de tester la validité de ces modèles sur le terrain en précisant certains paramètres au niveau régional dans des situations d'infestation par une adventice dominante. Deux écueils sont à éviter : une spécialisation trop biologique des modèles, où les rela- 
tions de concurrence entre plante cultivée et mauvaise herbe seraient étudiées hors de la réalité agronomique, et une orientation trop économique, où seraient négligées la relation entre le niveau d'infestation adventice et le rendement, base d'étude des seuils économiques annuels et pluriannuels de nuisibilité.

La détermination des seuils économiques pluriannuels de nuisibilité demeure actuellement très imprécise en raison du manque de données expérimentales concernant la dynamique des populations et servant à prédire leur taille à partir des potentiels semenciers. La principale limitation à l'utilisation des seuils biologiques de nuisibilité demeure la mise en œuvre de méthodes d'évaluation de la densité ou de la biomasse adventice à un moment précis, toutes très contraignantes en temps pour un agriculteur. II faut espérer que dans les prochaines années des méthodes d'étude de structure spatiale des adventices, avec analyse d'images traitée par ordinateur, seront mises au point. Ainsi la fiabilité des modèles sera améliorée, qu'il s'agisse de modèles prévisionnels ou de modèles de simulation.

\section{Remerciements}

Je remercie G. Barralis pour sa contribution critique au manuscrit ainsi que P. Jacquard, J. Maillet et B.J. Post pour leurs suggestions en tant que lecteurs.

\section{Références}

Aarts H.F.M. (1986) A computerized model for predicting changes in a population of Galium aparine. Proc. E.W.R.S. Sympos., Econ. Weed Control, Hohenheim (RFA), 277-284

Aarts H.F.M. \& De Visser, C.L.M. (1985) A management information system for weed control in winter wheat. Proc. british Crop Protect. Conf., Weeds 2, 679-686

Abd Elfatah H. (1987) Une approche fonctionnelle de la compétition entre espèces adventices et espèces cultivées / Application au cas de Solanum nigrum L. et de la tomate Lycopersicon esculentum Mill. Thèse Université des Sciences et Techniques du Languedoc, Montpellier II

Alabouvette C., Couteaudier Y. \& Louvet J. (1983) Importance des phénomènes de compétition nutritive dans l'antagonisme entre microorganismes. In: Les Antagonismes Microbiens, 24e Coll. SFP, Bordeaux, 26-28 mai 1983

Ammon H.U. (1988) Do actual thresholds favour the build-up of herbicide resistance in weed populations ? In : Weed Control in Vegetable Productions (R. Cavalloro \& A. El Titi, eds), Balkema, Rotterdam

Andersson B. (1986) Influence of crop density and spacing on weed competition and grain yield in wheat and barley. Proc. E.W.R.S. Sympos., Econ. Weed Control, Hohenheim (RFA), 121-128
Auld B.A. \& Tisdell C.A. (1985) Biological weed control-equilibria models. Agric. Ecosyst. Environm. $13,1-8$

Auld B.A. \& Tisdell C.A. (1986) Economic threshold/critical density models in weed control. Proc. E.W.R.S. Sympos., Econ. Weed Control, Hohenheim (RFA), 261-268

Auld B.A., Mentz K.M. \& Tisdell C.A. (1987) Weed Control Economics. Academic Press, London

Baggiolini M. (1977) L'utilisation du seuil de tolérance dans la lutte antiparasitaire en arboriculture. Rev. suisse Vitic. Arboric. Hortic. 9, 195-204

Balschun H. \& Jacob F. (1972) Über die zwischenartliche Konkurrenz von Linum usitatissimum L. und Camelina Arten. Flora 161, 129-172

Barralis G. \& Chadoeuf R. (1987) Potentiel semencier des terres arables. Weed Res. 27, 417-424

Barralis G. \& Chadoeuf R. (1988) Relations entre flore potentielle et flore réelle des champs cultivés. C.R. 8 e Coll. Intern. Biol. Ecol. Syst. Mauvaises Herbes, Dijon, $1 / 2,43-52$

Barralis G. \& Marnotte P. (1980) Contribution à l'étude de la concurrence entre plante cultivée et mauvaises herbes. C.R. 6e Coll. Intern. Ecol. Biol. Syst. Mauvaises Herbes, Montpellier, 2, 443-450

Beer E. (1986) Praktische Anwendung von Schadensschwellen in Winterroggen. Proc, E.W.R.S. Sympos. Econ. Weed Control., Hohenheim (RFA), 361-370

Begon M., Harper J.L. \& Townsend C.R. (1987) Ecology, Individuals, Populations and Communities. Blackwell, Oxford

Blackshaw R.P. (1986) Resolving economic decisions for the simultaneous control of two pests, diseases or weeds, Crop Protect. 5, 93-99

Bleasdale J.K.A. (1959) Studies on plant competition. In: The Biology of Weeds (J.L. Harpes, ed) Blackwell, Oxford, pp. 133-142

Bleiholder H. \& Nuyken W. (1986) Neue Ansätze zur Darstellung und Interpretation des Zusammenhanges zwischen dem Deckungsgrad der Unkräuter und dem Ertrag von Getreide. Proc. E.W.R.S. Sympos. Econ. Weed Control. Hohenheim (RFA), 61-68

Bode H.R. (1958) Beiträge zur Kenntnis allelopatischer Erscheinungen bei einigen Juglandaceen. Planta 51, 440-480

Borner H. (1968) Gegenseitige Beeinflussung höherer Pflanzen. In: Handbuch der Pflanzenkrankheiten (P. Sorauer, ed) Parey, Berlin, pp. 97-160

Branthome X., Caussanel J.P. \& Maillet J. (1986) Etude des effets de la concurrence de Solanum sp. sur tomates en semis direct. C.R. 13e Conf. Columa, Versailles, 3, 153-164

Buchanan G.A. (1977) Weed biology and competition. In: Research Methods in Weed Science (B. Truelove, ed) Southern Weed Science Society, Auburn University, Alabama, pp. 25-41

Burgos-Leon W. (1975) Phytotoxicité induite par les résidus de récolte de Sorghum vulgare dans les sols sableux de l'Ouest africain, origine et méthode biologique de détoxication des sols. Thèse 3 e Cycle, Agronomie, Univ. Nancy 1, UER STCM

Caputa J. (1948) Untersuchungen über die Entwicklung einiger Gräser und Kleearten in Reinsaat und Mischung. Landwirtsch. Jahrb. Schweiz 10, 849 
Carlson H.L. \& Hill J.E. (1985) Wild oats (Avena fatua) competition with spring wheat: plant density effects. Weed Sci. 33, 176-181

Caussanel J.P. (1979) Méthodes d'étude et d'estimation de la concurrence entre plantes cultivées et mauvaises herbes annuelles. C.R. 10e Conf. Columa, Paris, 4, 1191-1204

Caussanel J.P. \& Barralis G. (1973) Les phénomèries de concurrence entre végétaux. C.R. $4^{\ominus}$ Coll. Intern. Ecol. Biol. Mauvaises Herbes. Columa, Marseille, 202238

Caussanel J.P. \& Kunetsch G. (1979) Etude qualitative et quantitative des inhibiteurs de croissance présents dans les exsudats de racines de chénopode blanc (Chenopodium album L.) au début de sa floraison, en culture hydroponique et sous conditions contrôlées. $Z$. Pflanzenphysiol. 93, 229-243

Caussanel J.P., Barralis G., Vacher C., Fabre E., Morin C. \& Branthome X. (1986) La variation des seuils de nuisibilité des mauvaises herbes : résultats expérimentaux. Perspect. agric. 109, 22-28

Caussanel J.P., Kafiz B. \& Carteron, A. (1988) Analyse expérimentale des effets de concurrence d'une grarninée adventice dans un blé de printemps en relation avec le désherbage. Weed Res. 28, 309-322

Chadoeuf-Hannel R. (1985) La dormance chez les semences de mauvaises herbes. Agronomie 5, 761 772

Chancellor R. \& Peters C.B. (1976) Competition between wild oats and crops. In: Wild Oats in World Agriculture (D. Price-Jones, ed), A.R.C., London, pp. 99-112

Chessel D. (1978) La description non paramétrique de la dispersion spatiale des individus d'une espèce. In: Biométrie et Ecologie (J.M. Legay \& R. Tomassone, ed), INRA, pp. 45-135

Chiang H.C. (1979) A general model of the economic threshold of pest populations. FAO Plant Protection Bull. 27, 71-73

Chiarappa L. (1971) Crop Loss Assessment Methods. FAO Manual on the evaluation and prevention of losses by pests, diseases and weeds. FAO and CAB, Farnham Royal, UK

Chiarappa L. (1981) Crop Loss Assessment Methods., Suppl. 3., FAO and CAB, Farnham Royal, UK

Chisaka H. (1977) Weed damage to crops: yield loss due to weed competition In: Integrated Control of Weeds (D. Fryer \& S. Matsunatkra, eds) Univ. Tokyo Press, Tokyo, 1-16

Coder K.D. (1983) Seasonal changes of juglone potential in leaves of black walnut (Junglans nigra L..). J. chemical Ecol. 9, 8, 1203-1212

Cousens R. (1985a) A simple model relating yield loss to weed density. Ann. Appl. Biol. 107, 239-252

Cousens R. (1985b) An empirical model relating crop yield to weed and crop density and a statistical comparison with other models. J. Agric. Sci. 105, 513-521

Cousens R.D. (1986) The use of population models in the study of the economics of weed control. Proc. E.W.R.S. Sympos. Econ. Weed Control, Hohenheim (RFA), 269-276

Cousens R., Pollard F. \& Denner A.P. (1985) Competition between Bromus sterilis and winter cereals. Aspects appl. Biol. 9, 67-74

Cousens R., Wilson B.J. \& Cussans G.W. (1985) To spray or not to spray: the theory behind the practice. Proc. brit. Crop Protect. Conf., Weeds 2, 671-678
Cousens R., Doyle C.J., Wilson B.J. \& Cussans G.W. (1986) Modelling the economics of controlling Avena fatua in winter wheat. Pestic. Sci. 17, 1-12

Cousens R., Moss S.R., Cussans G.W. \& Wilson B.J. (1987) Modelling weed populations in cereals $I n$ : Reviews of Weed Science, vol. 3. Weed Sci. Soc. Am., Champaign, Illinois

Covarelli G. \& Tei F. (1984) Compétition entre tournesol et Sinapis arvensis L. à différents niveaux d'infestation. C.R. 6e Coll. Intern. Ecol. Biol. Syst. Mauvaises Herbes, Montpellier, 1, 323-330

Cramer H.H. (1967) La protection des plantes et les récoltes dans le monde. Pflanzenschutz Nachr., Bayer Cussans G.W. \& Moss S.R. (1982) Population dynamics of annual grass weeds. Proc. brit. Crop Protect. Sympos. Decision Making in the Practice of Crop Protection, 91-98

Cussans G.W., Cousens R.D. \& Wilson B.J. (1986) Thresholds for weed control-the concepts and their interpretation. Proc. E.W.R.S. Sympos., Econ. Weed Control, Hohenheim (RFA), 253-260

Darmency H. (1987) Polymorphisme et sélection pour la résistance aux herbicides chez les mauvaises herbes. Thèse Doct. Univ. Paris Sud, Orsay

Dawson J.H. (1986) The concept of period thresholds. Proc E.W.R.S. Sympos., Econ. Weed Control, Hohenheim (RFA), 327-331

Debaeke P. (1988) Modélisation de l'évolution à long terme de la flore adventice. Il : application à trois dicotylédones annuelles en un site donné. Agronomie 8, 767-777

Deleuil G. (1950) Mise en évidence de substances toxiques pour les thérophytes dans les associations du Rosmarino-Ericion. C.R. Acad. Sci 230, 1362-1364

Dew D.A. (1972) An index of competition for estimating crop loss due to weeds. Can. J. Plant Sci 52, 921927

Doyle C.J., Cousens R. \& Moss S.R. (1986) A model of the economics of controlling Alopecurus myosuroides Huds. in winter wheat. Crop Protect. 5, 143-150 Duke S.O. (1986) Naturally occurring chemicals compounds as herbicides $\mathrm{In}$ : Reviews of Weed Science, vol. 2. Weed Sci. Soc. Am., Champaign, Illinois, pp. 15-44

Eggers T. (1975) Konkurrenz der Unkraüter untereinander. Z. Pflanzenkr. Pflanzenschutz 7, 87-94

Eggers T. \& Niemann P. (1980) Zum Begriff des Unkrauts aus phytomedizinischer Sicht und über Schadsschwellen bei der Unkrautbekämpfung, Gesunde Pflanz 32, 1-7

Egley G.H. (1986) Stimulation of weed seed competition in soil. In: Reviews of Weed Science, vol. 2, Weed Sci. Soc. Am., Champaign, Illinois, pp. 67-89

Ellern S.J., Harper J.L. \& Sagar G.R. (1970) A comparative study of the distribution of the roots of Avena fatua and $A$. strigosa in mixed stands using a $14 \mathrm{C}$ labelling technique. J. Ecol. 58, 865-868

Elliott J.G. (1980) The economic significance of weeds in the harvesting of grain. Proc. british Crop Protect. Conf. Weeds 3, 787-797

Fay P.K. \& Duke W.B. (1977) An assessment of allelopathic potential in Avena germ plasm Weed Sci. 25 , 224-228

Firbank L.G., Mortimer A.M. \& Putwain P.D. (1985) Bromus sterilis in winter wheat: a test of a predictive population model. Aspects Appl. Biol. 9, 59-66 
Fuerst E.P. \& Putnam A.R. (1983) Separating the competitive und allelopathic components of interference: theoretical principles. J. chemical Ecol. 9, 937-944

Gabor W.E. \& Veatch C. (1981) Isolation of a phytotoxin from quackgrass (Agropyron repens) rhizomes. Weed Sci. 29, 155-159

Garburg W. \& Heitefuss R. (1975) Untersuchungen zur Ermittlung von ökonomischer Schadensschwellen und Bekampfungsschwellen von Unkräuten in Getreide. Z. Pflanzenkr. Pflanzenschutg. 7, 71-77

Gasquez J. (1984) Approche génétique des mauvaises herbes : variabilité intraspécifique, évolution, résistance. Schweiz. Landwirtsch. Forsch. 23, 77-88

Gerowitt B. (1987) Unkrautbekämpfung nach Schadensschwellen im Wintergetreide. Überprüfung und Weiterentwicklung des Konzepts mit Hilfe einer bundesweiten Versuchsserie und Erarbeitung eines Computergestützten Entscheidungsmodells. Thèse Univ., Göttingen (RFA)

Glauninger J. \& Holzner W. (1982) Interference between weeds and crops: a review of litterature $\ln :$ Biology and Ecology of Weeds (W. Holzner \& M. Numata, eds) W. Junk, La Haye, pp. 149-159

Gressel J. (1987) Appearance of single and multigroup herbicide resistances and strategies for their prevention. Proc. british Crop Protect. Conf. Weeds 4, 479488

Grodzinsky A.M. (1982) Prospects for allelopathy study and use in crop production. Rol. allelopatii $v$ rastenievodstve. Sci. Coll. Kiev Nauk. dumka, 3-14 (en russe)

Grodzinsky A.M. \& Gaidamak V.M. (1971) Allelopathic influence of woody plants on herbaceous ones in the Ukrainian forest-steppe region. Fiziol. Biokhim. Osn. Vzaimodejstviya Rasten. Fitotsenozkh, 3-11 (en russe)

Grummer G. (1961) The role of toxic substances in the inter-relationships between higher plants. In: Symp. Soc. Exp. Biol. Mechanisms in Biological Competition (F.L. Milthorpe, ed) Academic Press, New York, pp. 219-228

Grummer G. \& Beyer H. (1960) The influence exerted by species of Camelina on flax by means of toxic substances. In: The Biology of Weeds (J.L. Harper, ed) Academic Press, London, pp. 153-157

Guenzi W.D., MC Calla T.M. \& Norstadt F.A. (1967) Presence and persistence of phytotoxic substances in wheat, oats, corn and sorghum residues. Agron. J. 59, 163-165

Guillemenet R. (1972) Etude de la concurrence exercée par le vulpin des champs à l'égard du blé tendre d'hiver. Phytoma 24, 9-12

Guy P. (1965) La compétition intraspécifique chez les plantes fourragères. Fourrages $F r .22,12-25$

Hagemeister $H$. \& Heitefuss R. (1986) Einfluss einer unterschiedlich intensiven Unkrautbekämpfung im Getreide auf den Samenvorrat im Boden und die Verunkrautung in Folgekulturen. Proc. E.W.R.S. Sympos., Econ. Weed Control, Hohenheim (RFA), 393-398

Haizel K.A. \& Harper J.L. (1973) The effects of density and the timing of removal on interference between barley, white mustard, and wild oats. J. appl. Ecol. 10, 2331

Hakansson S. (1983) Competition and Production in Short-lived Crop-weed Stands. Sveriges Lantbruksuniversitet, Institutionen för växtodling, Rapport 127
Hakansson S. (1986) Competition between crops and weeds-influencing factors, experimental methods and research needs. Proc. E.W.R.S. Sympos. Econ. Weed Control, Hohenheim (RFA) 49-68

Harlan J.R. (1982) Relationships between weeds and crops. In: Biology and Ecology of Weeds (W. Holzner \& M. Numata, eds). W. Junk, La Haye, pp. 91-96

Harper J.L. (1977) Population Biology of Plants. Academic Press, London

Heitefuss R., Gerowitt B. \& Wahmhoff W. (1987) Development and implementation of weed economic thresholds in the F.R. Germany. Proc. british Crop Protect. Conf. Weeds 4, 1025-1034

Jacquard P. (1968) Manifestation et nature des relations sociales chez les végétaux supérieurs. Acta Oecol. Oecol. Plant. 3, 137-168

Jacquard P. (1975) Concurrence intraspécifique et potentialités de rendement. Ann. Amélior. Plantes 25, 3-24

Jacquard P. (1980) Aspects démographiques, génétiques et fonctionnels de la biologie des populations de mauvaises herbes. C.R. 6e Coll. Intern. Ecol. Biol. Syst. Mauvaises Herbes, Montpellier, 2, 273-286

Jacquard P. (1982) Dynamique des relations de concurrence interspécifiques, interpopulations et intrapopulations : modèles prévisionnels et de simulation. Acta Oecol. Oecol. gen. 3, 183-215

Jensen P.K. (1985) A review of yield responses to weed control in one thousand spring barley experiments. Proc. british Crop Protect. Conf. Weeds 2, 687692

Kees H. (1986) Einfluss zehnjähriger Unkrautbekämpfung mit 4 unterschiedlichen Intensitätsstufen unter Berücksichtigung der wirtschaftlichen Schadensschwelle auf Unkrautflora und Unkrautsamenvorrat im Boden. Proc. E.W.R.S. Sympos., Econ. Weed Control, Hohenheim (RFA), 399-406

Keisling T.C., Olivier, L.R., Crowley, R.H. \& Baldwin, F.L. (1984) Potential use of response surface analysis for weed management in soybeans (Glycine max). Weed Science 32, 552-557

Kemmer A. \& Hurle K. (1984) Überprüfung wirtschaftlicher Schadensschwellen am Beispiel Ackerfuchsschwanz in Winterweizen. Z. Pflkrankh. Pflschutz 10, 175180

Kheddam M. (1988) Contribution à l'étude de la répartition spatio-temporelle et de la nuisibilité des mauvaises herbes dans une culture de maïs. Thèse Doct. Ing. E.N.S.A.R., Univ. Rennes I

King L.J. (1966) Injurious interactions of weeds and crop plants. In: Weeds of the World. Biology and Control (L. J. King, ed), Leonard Hill, London, pp. 243259

Koch W. (1967) Untersuchungen zur Konkurrenzwirkung von Kulturpflanzen und Unkraütern aufeinander. II. Schadwirkung von Samenunkraütern auf Getreide. Weed Res. 7, 1, 22-28

Koch W., Beshir M.E. \& Unterladstatter R. (1982) Crop loss due to weeds. FAO Plant Protect. Bull. 30, 103111

Koch W. \& Walter H. (1983) The effects of weeds in certain cropping systems. Proc. 10th Intern. Cong. Plant Protect, Brighton, UK, pp. 90-97

Kolbe W. (1977) Mehrjährige Untersuchungen über Beziehungen zwischen Unkraut-Deckungsgrad und 
Mehrertrag bei chemischer Unkrautbekämpfung (19671976). Z. Pflanzenkr. Pflanzenschultz 8, 59-67

Kranz E. \& Jacob F. (1977) Zur Mineralstoff-Konkurrenz zwischen Linum und Camelina Arten. II. Aufnahme von ${ }^{32}$ P-Phosphat und ${ }^{86}$ Rubidium. Flora 166, 505516

Kropff M.J. (1988) Simulation of crop weed competition. In: Weed Control in Vegetable Production (R. Caval-loro \& A. El Titi, eds), Balkema, Rotterdam, pp. 73-83

Leather G.R. (1983) Sunflowers (Helianthus annuus) are allelopathic to weeds. Weed Sci. 31, 37-42

Le Clerg E.L. (1971) Field experiments for assessment of crop losses. In: Crop Loss Assessment Methods. FAO Manual on the Evaluation and Prevention of Losses by Pests, Diseases and Weeds (L. Chiarappa, ed). FAO and CAB, Farnham Royal, UK, pp. 21-32

Legay J.M. \& Debouzie D. (1985) Introduction à une Biologie des Populations. Masson, Paris

Lemée G. (1967) Facteurs biotiques. In: Précis de Biogéographie. (G. Lemée, ed), Masson, Paris, pp. 171-197

Lemee G. (1978) Facteurs biotiques In: Précis d'Ecologie Végétale (G. Lemee, ed). Masson, Paris, pp. 143-166

Liebl R.A. \& Worsham, D. (1983) Inhibition of pitted morning glory (Ipomoea lacunosa $L$.) and certain other weed species by phytotoxic components of wheat (Triticum aestivum L.) straw. J. chem. Ecol. 9, 1027-1043

Longchamp R. (1977a) La nuisibilité des mauvaises herbes. C.R. 9 e Conf. Columa, Paris, 4, 970-975

Longchamp R. (1977b) Seuils de nuisibilité des mauvaises herbes. Phytoma 29, 288, 7-11

Loubaresse J.P., Mouillac A. \& Lejeune F. (1975) Seuils de nuisibilité des folles avoines dans les céréales. C.R. 8e Conf. Columa, Paris, 401-410

Lovett J.V. \& Jackson H.F. (1980) Allelopathic activity of Camelina sativa (L.) Crantz in relation to the phyllosphere bacteria. New Phytol. 86, 273-277

Madeira J., Dordio M.F. Mira, R.S. \& Lopes S. (1984) Population level and concurrence of wild oats (Avena sterilis, ssp sterilis) in wheat fields in Portugal, 461468. C.R. 3e Symp. sur les mauvaises herbes et le désherbage dans le Bassin Méditerranéen; E.W.R.S., Oeiras, Portugal, pp. 461-468

Maillet J. \& Abd Elfatah H. (1983) Etudes préliminaires sur la concurrence entre Solanum nigrum ssp eunigrum L. (morelle noire) et Lycopersicon esculentum Mill. (tomate) en culture repiquée. Weed Res. 423, 217-219

Malcolm W.M. (1966) Biological interactions. Bot. Rev. 32, 243-254

Manlove R.J., Mortimer A.M. \& Putwain A.D. (1982) Modelling wild oats populations and their control. Proc. brit. Crop Protect. Conf. Weeds 749-756

Marra M.C. \& Carlson G.A. (1983) An economic threshold model for weeds in soybeans (Glycine max). Weed Sci 31, 604-609

Marshall E.J.P. (1987) Using decision thresholds for the control of grass and broad-leaved weeds at the Boxworth E.H.F. Proc. brit. Crop Protect. Conf. Weeds 1059-1066

Martin P. (1957) Die Abgabe von organischen Verbindungen insbesondere von Scopoletin aus den Keim- wurzeln des Hafers. Z. Bot. 45, 475-506

Martin P. \& Rademacher B. (1960) Studies on the mutual influences of weeds and crops. In: The Biology of Weeds (J.L. Harper, ed). Blackwell, Oxford, pp; 143-152

Mead R. \& Pike D.J. (1975) A review of response surface methodology from a biometric viewpoint. Biometrics 31, 803-851

Medd R.W., Auld B.A., Kemp D.R. \& Murison R.D. (1985) The influence of wheat density and spatial arrangement on annual ryegrass, Lolium rigidum Gaudin, competition, Austr. J. Agric. Res. 36, 361-371

Milne A. (1961) Definition of competition among animals. In: Mechanisms in Biological Competition (F.L. Milthorpe, ed) Academic Press, New York, pp. 40-61

Milthorpe F.L. (1961) The nature and analysis of competition between plants of different species. $[n$ : Mechanisms in Biological Competition (F.L. Milthorpe, ed). Academic Press, New York, pp. 330-335

Mondragon G. (1988) Contribution a l'étude de seuils de nuisibilité biologiques de quelques espèces d'avoine dans une culture de blé. Thèse Doct.-Ingén., ENSAR, Univ. Rennes I

Montegut J. (1975) Ecologie de la germination des mauvaises herbes. In: La Germination des Semences (R. Chaussat \& Y. Le Deunff, eds) Gauthier-Villars, Paris, pp. 191-217

Morin C., Caussanel J.P., Capdeboscq J.B., Gripon L. \& Taillet D. (1988) Etude de la période de concurrence d'une population résistante aux triazines de Solanum nigrum L. dans le maïs. C.R. 8 e Coll. inter. Biol. Ecol. Syst. des Mauvaises Herbes, Dijon, 2, 549-561

Mortimer A.M. (1983) On weed demography. In: Recent Advances in Weed Research (W.W. Fletches, ed) C.A.B., Farnham Royal, London, pp. 3-40

Mortimer A.M. \& Putwain P.D. (1984) The prediction of weed infestations: concepts and approaches. EPPO Bull. 14, 439-446

Muller C.H. (1966) The role of chemical inhibition (allelopathy) in vegetational composition. Bull. Torrey bot. Club 93, 332-351

Muller C.H., Lorber P. \& Haley B. (1968) Volatile growth inhibitors produced by Salvia leucophylla : effect on seedling growth and respiration. Bull. Torrey bot. Club 5, 415-422

Mumford J. (1982) Perceptions of losses from pests of arable crops by some farmers in England and New Zealand. Crop Protection 1, 283-288

Musselman L.J. (1982) Parasitic weeds of arable land. In: Biology and Ecology of Weeds (W. Holzner \& M. Numata, eds) W. Junk, La Haye, pp. 175-185

Naylor R.E.L. (1970) The prediction of blackgrass infestations. Weed Res. 10, 296-299

Neururer H. (1975) Weitere Erfahrungen in der Beurteilung der tolerierbaren Verunkräutungsstärke. Z. Pflanzenkrankh. Pflanzenschutz 7, 63-69

Neururer H. (1976) Ökonomische Schadensschwelle und tolerierbare Verunkrautsstärke in der Unkrautbekämpfung. Landforstwirtsch. Forsch. Österreich 7, 143-153

Niemann P. (1986) Mehrjährige Anwendung des Schadensschwellenprinzips bei der Unkrautbekämpfung auf einem Landwirtschaftlichen Betrieb. Proc. E.W.R.S. Sympos. Econ. Weed Control, Hohenheim (RFA), 385392 
Nieto J.H., Brando M.A. \& Gonzales J.T. (1968) Critical periods of the crop growth cycle for competition from weeds. PANS 14, 159-166

Numata M. (1956) Experimental studies on early stages of secondary succession. Jpn. J. Ecol. 6, 62-66 O'Donovan J.T., St Remy E.A., O'Sullivan P.A., Dew D.D. \& Sharma A.K. (1985) Influence of the relative time of emergence of wild oats (Avena fatua) on yield loss of barley (Hordeum vulgare) and wheat (Triticum aestivum). Weed Sci. 33, 498-503

Odum E.P. (1959) Fundamentals of Ecology. W.B. Saunders, Philadelphia

Olivier J.M. (1983) Les organismes antagonistes d'agents pathogènes. In: C.R. Journées d'études 4 et 5 mai 1983. Faune et Flore Auxiliaires en Agriculture. Acta, Paris, pp. 145-163

O'Sullivan P.A., Kossatz V.C., Weiss G.M. \& Ng Dew D.A. (1982) An approach to estimate yield loss of barley due to Canada thistle. Can. J. Plant Sci. 62, 725731

Osvald H. (1948) Toxic exudates from the roots of Agropyron repens (couch grass). J. Ecol. 36, 192-193

Peters N.C.B. (1972) Methods for evaluating weed competition using systems of hand-weeding or hoeing. Proc. 11th brit. Weed Control Conf., pp. 116-118

Peters N.C.B. (1985) Competititve effects of Avena fatua $L$. plants derived from seeds of different weights. Weed Res. 25, 67-77

Pinstrup-Andersen P., De Londono N. \& Infante M. (1976) A suggested procedure for estimating yield and production losses in crops. PANS 22, 359-365

Poole M.L. \& Gill G.S. (1987) The use of weed density, crop yield relationships for predicting yield losses in the field. Proc. british Crop Protect. Conf. Weeds, 4, 945-954

Putnam A.R. (1985) Weed allelopathy. In: Weed Physiology (S.O. Duke, ed) CRC Press, Boca Raton, Florida, pp. 131-155

Putnam A.R. \& Duke W.B. (1974) Biological suppression of weeds : evidence for allelopathy in accessions of cucumbers. Science 185, 370-372

Putnam A.R. \& Duke W.B. (1978) Allelopathy in agroecosystems. Annu. Rev. Phytopathol 16, 431-451

Radosevich S.R. \& Holt J.S. (1984) Weed Ecology. Implications for Vegetation Management. Wiley Interscience, New York

Rauber R. (1977) Kurz-und langfristige Auswirkungen bei der Bekämpfung von Flughafer (Avena fatua $L$.) in Sommergerste. Z. Pflanzenkr. Pflanzenschutz 8, 107 117

Rauber R. \& Koch W. (1975) Zur Populationdynamik des Flughafers (Avena fatua L.) unter dem Aspekt der langfristigen Befallsprognose. Proc. E.W.R.S. Sympos., Status, Biol. and Control Grass Weeds in Europe, 113-123

Rauber R., Kemmer A. \& Koch W. (1980) Untersuchungen zur Ermittlung einer Bekämpfungsschwelle für Ackerfuchsschwanz (Alopecurus myosuroïdes Huds) in Winterweizen. Gesunde Pflanz 32, (8), 177181

Reeves T.G. (1976) Effect of annual ryegrass (Lolium rigidum Gaud.) on yield of wheat. Weed Res 16, 57-63

Regnault Y. \& Pouzet A. (1983) Etude du désherbage complémentaire du colza. C.R. 12e Conf. Columa, Paris, 2, 193-200
Rice E.L. (1971) Some possible roles of inhibitors in oldfield succession. In: Biochemical Interactions Among Plants Ed. US Nat. Comm. for IBP, Nat. Acad. Sci. Washington, pp. 120-132

Rice E.L. (1974) Allelopathy. Academic Press. New York

Roberts E.H. (1972) Dormancy: a factor affecting seed survival in the soil. In: Viability of Seeds (E.H. Roberts, ed) Chapman \& Hall, London, pp. 321-359

Roberts H.A. (1981) Seed banks in soil. Adv. appl. Biol 6, 1-55

Sagar G.R. (1968) Factors affecting the outcome of competition between crops and weeds. Proc. 9th British Weed Control Conf., 1157-1162

Sagar G.R. (1970) Factors controlling the size of plant populations. Proc. 10th Brit. Weed Control Conf., 965979

Sagar G.R. \& Mortimer A.M. (1976) An approach to the study of the population dynamics of plants with special reference to weeds. Ann. appl. Biol. 1, 1-47

Schumacher W.J., Thill D.C. \& Lee G.A. (1983) Allelopathic potential of wild oats (Avena fatua) on spring wheat (Triticum aestivum) growth. J. chem. Ecol. 9, 1235-1245

Schweizer E.E. \& Bridge L.D. (1982) Sunflower (Helianthus annuus) and velvetleaf (Abutilon theophrasti). Interference in sugarbeets (Beta vulgaris). Weed Sci. 30, 514-530

Sebillotte M. (1967) Compétition entre blé et graminée adventice. Rôle des rotations culturales C.R. Acad. Agric. Fr. 13, 74-88

Sill Webster H. Jr. (1982) Biological control by antagonism. In: Plant Protection, an Integrated Interdisciplinary Approach. lowa State Univ. Press, Ames, lowa, pp. 168-184

Spitters C.J.T. \& Aerts R. (1983) Simulation of competition for light and water in crop weed associations. Aspects appl. Biol. 4, 467-483

Spitters C.J.T. \& Van Den Bergh J.P. (1982) Competition between crop and weeds: a system approach. In: Biology and Ecology of Weeds (W. Holzner \& $M$. Numata, eds) W. Junk, La Haye, pp. 137,140

Tait E.J. (1977) A method for comparing pesticide usage patterns between farmers. Ann. appl. Biol. 86, 229-240

Tauscher B. (1988) Allelochemicals, eine interdisziplinäre Herausforderung. Z. Pflanzenkr. Pflanzenschutz 11, 15-31

Thioulouse J. (1985) Structures spatio-temporelles en biologie des populations d'insectes. Application à l'étude de l'altise du colza (Psyllioides chrysocephala L.): résultats méthodologiques et biologiques. Thèse de $3 \mathrm{~B}$ Cycle, Univ. Lyon I

Thioulouse J. (1987) Space-time structures in a winter rape pest population, Psyllioides chrysocephala (Col. Chrysomelidæ): methodological proposals and biological interpretations. J. appl. Ecol. 24, 435-450

Thurston J.M. (1982) Wild oats, as successful weeds. In: Biology and Ecology of Weeds (W. Holzner \& M. Numata, eds) W. Junk, La Haye, pp. 191-199

Tissut M. \& Séverin F. (1984) Désherbage, mise en œuvre des herbicides et conséquences. In: Plantes, Herbicides et Désherbage: Bases Scientifiques et Techniques (M. Tissut \& F. Séverin, eds), Acta Paris, pp. 179-230 
Trenbath B.R. (1976) Plant interactions in mixed crop communities. In: Multiple Cropping. Am. Soc. Agronomy, pp. 129-169

Tukey H.B. (1969) Implications of allelopathy in agricultural plant science. Bot. Rev 35, 1-16

Vacher C. \& Fabre E. (1986) Contribution à l'étude de la période de concurrence d'Alopecurus myosuroïdes (L.) Huds. et de la nuisibilité de Lolium multiflorum $L$. Proc. E.W.R.S. Sympos., Econ. Weed Control, Hohenheim (RFA) 129-136

Wahmhoff W. (1986) Erfahrungen mit der praktischen Anwendung von Schadensschwellen bei der Unkrautbekämpfung in Getreide. Proc. E.W.R.S. Sympos., Econ. Weed Control, Hohenheim (RFA), 379-384

Wahmhoff W. \& Heitefuss R. (1984) Der Einfluss der Berücksichtigung von Schadensschwellen für Unkraüter in Wintergerste auf Kornertrag, Qualität des Erntegutes, Erntetechnik und Wirtschaftlichkeit der Unkrautbekämpfung. Z. Pflanzenkr. Pflanzenschutz 10, 137-148

Walker P.T. (1983) Crop losses: the need to quantify the effects of pests, diseases and weeds on agricultural production. Agric. Ecosyst. Environment 9, 119-158

Watkinson A.R. (1981) Interference in pure and mixed populations of Agrostemma githago. J. appl. Ecol. 18, 967-976

Weaver S. (1984) Critical period of weed competition in three vegetable crops in relation to management practices. Weed Res. 24, 317-325

Welbank P.J. (1963) Toxin production during decay of Agropyron repens (couch grass) and other species. Weed Res. 3, 205-214

Whittaker R.H. (1970) The biochemical ecology of higher plants. In: Chemical Ecology (E. Sondheimer \& J.B. Simeone, eds) Academic Press, New York, pp. 43-70

Wiese A.F. (1971) Method N62. In: Crop Loss Assessment Methods. FAO Manual on the Evaluation and Prevention of Losses by Pests, Diseases and Weeds (L. Chiarappa, ed) FAO \& CAB, Farnham Royal, UK
Willey R.W. \& Heath S.B. (1969) The quantitative relationships between plant population and crop yield. Adv. Agron. 21, 281-321

Williams C.S. \& Hayes, R.M. (1984) Johnsongrass (Sorghum halepense) competition in soybeans (Glycine max). Weed Sci. 32, 498-501

Wilson B.J. (1986) Yield responses of winter cereals to the control of broad-leaved weeds. Proc. E.W.R.S. Sympos., Econ. Weed Control Hohenheim (RFA), 7582

Wilson B.J. \& Cussans G.W. (1983) The effect of weeds on yield and quality of winter cereals in the U.K. Proc. 10th Intern. Congr. Plant Protect. 1, 121

Wilson R.E. \& Rice E.L. (1968) Allelopathy as expressed by Helianthus annuus and its role in old-field succession. Bull. Torrey bot. Club 95, 432-448

Wilson B.J., Cousens R. \& Cussans G.W. (1984) Exercises in modelling populations of Avena fatua $L$. to aid strategic planning for the long term control of this weed in cereals. C.R, 7e Coll. intern. Ecol. Biol. Syst. Mauvaises Herbes, Paris, 1, 287-294

Wimschneider W. \& Bachthaler G. (1979) Untersuchungen über die Lichtkonkurrenz zwischen Avena fatua L. und verschiedenen Sommerweizensorten. Proc. E.W.R.S. Sympos. Influence of different factors on the development and control of weeds. Mayence, pp. 249-256

Wit C.T. (1960) On competition. Versl. Landbouwkd. Onderz. Rijkslandbouw. proefstat. 66, 1-82

Wojewedka A. \& Morin C. (1983) Evaluation de la concurrence exercée par les amaranthes (Amaranthus retroflexus) résistantes aux triazines : recherche d'un seuil de nuisibilité dans le maïs. C.R. $12^{\ominus}$ Conf. Columa 2, 64-73

Zimdahl R.L. (1980) Weed Crop Competition: a Review. Int. Plant Protect. Center (IPPC), Corvallis, Oregon State Univ.

Zwerger P. \& Hurle K. (1988) Simulationstudien zum Einfluss von Fruchtfolge und Bekämpfungsmassnahmen auf die Verunkrautung $Z$. Pflanzenkr. Pflanzenschutz 11, 71-82 